\title{
A Polymer-Coated Rhodium/Diamine-Functionalized Silica for Controllable Reaction Switching in Enantioselective Tandem Reduction-Lactonization of Ethyl 2-Acylarylcarboxylates
}

\author{
Lingyu Kong, Junwei Zhao, Tanyu Cheng, Jingrong Lin, Guohua Liu*
}

Key Laboratory of Resource Chemistry of Ministry of Education, Shanghai Key Laboratory of Rare Earth Functional Materials, Shanghai Normal University, Shanghai, China. Tel: + 86 21 64322280; E-mail: ghliu@shnu.edu.cn.

\begin{tabular}{|c|c|c|}
\hline & Contents & Page \\
\hline Experimental & General and characterization and preparation & $\mathrm{S} 2$ \\
\hline Figure S1 & FT-IR spectra of 2 and catalyst 3. & $\mathrm{~S} 3$ \\
\hline Figure S2 & ${ }^{13} \mathrm{C}$ CP MAS NMR spectrum of its polymeric analog 3'. & S3 \\
\hline Figure S3 & $\begin{array}{l}\text { Melting point of catalyst } 3 \text { determined by differential scanning } \\
\text { calorimetry at a heating rate of } 30 \mathrm{~K} / \mathrm{min} \text {. }\end{array}$ & S4 \\
\hline Figure S4 & $\begin{array}{l}\text { Average hydrodynamic diameters distribution measurement for the } \\
\text { recycled catalyst } 3 \text { at indicated } 15^{\circ} \mathrm{C} \text { and } 35^{\circ} \mathrm{C}\end{array}$ & $\mathrm{S} 4$ \\
\hline Figure S5 & ${ }^{1} \mathrm{H}-\mathrm{NMR},{ }^{13} \mathrm{C}-\mathrm{NMR}$ and GC-MS of all chiral products. & $\mathrm{S} 10$ \\
\hline Figure S6 & $\begin{array}{l}\text { Asymmetric synthesis of phthalides in the enantioselectivite tandem } \\
\text { reduction-lactonization reaction of 2-acylarylcarboxylates. }\end{array}$ & $\mathrm{S} 32$ \\
\hline Table S1 & $\begin{array}{l}\text { Reusability of } 3 \text { using ethyl 2-(2-(4-bromophenyl)acetyl)benzoate as a } \\
\text { substrate }\end{array}$ & S43 \\
\hline Figure S7 & $\begin{array}{l}\text { Reusability of } 3 \text { using ethyl 2-(2-(4-bromophenyl)acetyl)benzoate as a } \\
\text { substrate }\end{array}$ & S43 \\
\hline
\end{tabular}




\section{Experimental}

\section{General}

All experiments, which are sensitive to moisture or air, were carried out under an $\mathrm{Ar}$ atmosphere using the standard Schlenk techniques. Acrylamide (Fisher Scientific, $\geqq 99.9 \%$, electrophoresis grade, $\leqq 0.002 \%$ acrylic acid), acrylonitrile (Acros, $>99 \%$ ), 2,2-azobisisobutyronitrile (AIBN), tetraethoxysilane (TEOS), $\left[\mathrm{Cp}^{*} \mathrm{RhCl}_{2}\right]_{2}$, and N-((S,S)2-amino-1,2-diphenylethyl)-4-vinylbenzenesulfonamide were purchased from SigmaAldrich Company Ltd. Compound of (S,S)-4-(trimethoxysilyl)ethyl)phenylsulfonyl-1,2diphenylethylenediamine and the $\mathrm{SiO}_{2}$-nanospheres were synthesized according to the reported literatures [(a) J. Mater. Chem., 2010, 20, 1970. (b) Langmuir, 2013, 29, 1010.].

\section{Characterization}

$\mathrm{Rh}$ loading amount in the catalyst was analyzed using an inductively coupled plasma optical emission spectrometer (ICP, Varian VISTA-MPX). Fourier transform infrared (FTIR) spectra were collected on a Nicolet Magna 550 spectrometer using KBr method. Scanning electron microscopy (SEM) images were obtained using a JEOL JSM-6380LV microscope operating at $20 \mathrm{kV}$. Transmission electron microscopy (TEM) images were performed on a JEOL JEM2010 electron microscope at an acceleration voltage of $220 \mathrm{kV}$. Solid state NMR experiments were explored on a Bruker AVANCE spectrometer at a magnetic field strength of $9.4 \mathrm{~T}$ with ${ }^{1} \mathrm{H}$ frequency of $400.1 \mathrm{MHz}$, and ${ }^{13} \mathrm{C}$ frequency of 100.5 MHz with $4 \mathrm{~mm}$ rotor at two spinning frequency of $5.5 \mathrm{kHz}$ and $8.0 \mathrm{kHz}$, TPPM decoupling is applied in the during acquisition period. ${ }^{1} \mathrm{H}$ cross polarization in all solid state NMR experiments was employed using a contact time of $2 \mathrm{~ms}$ and the pulse lengths of $4 \mu \mathrm{s}$. 


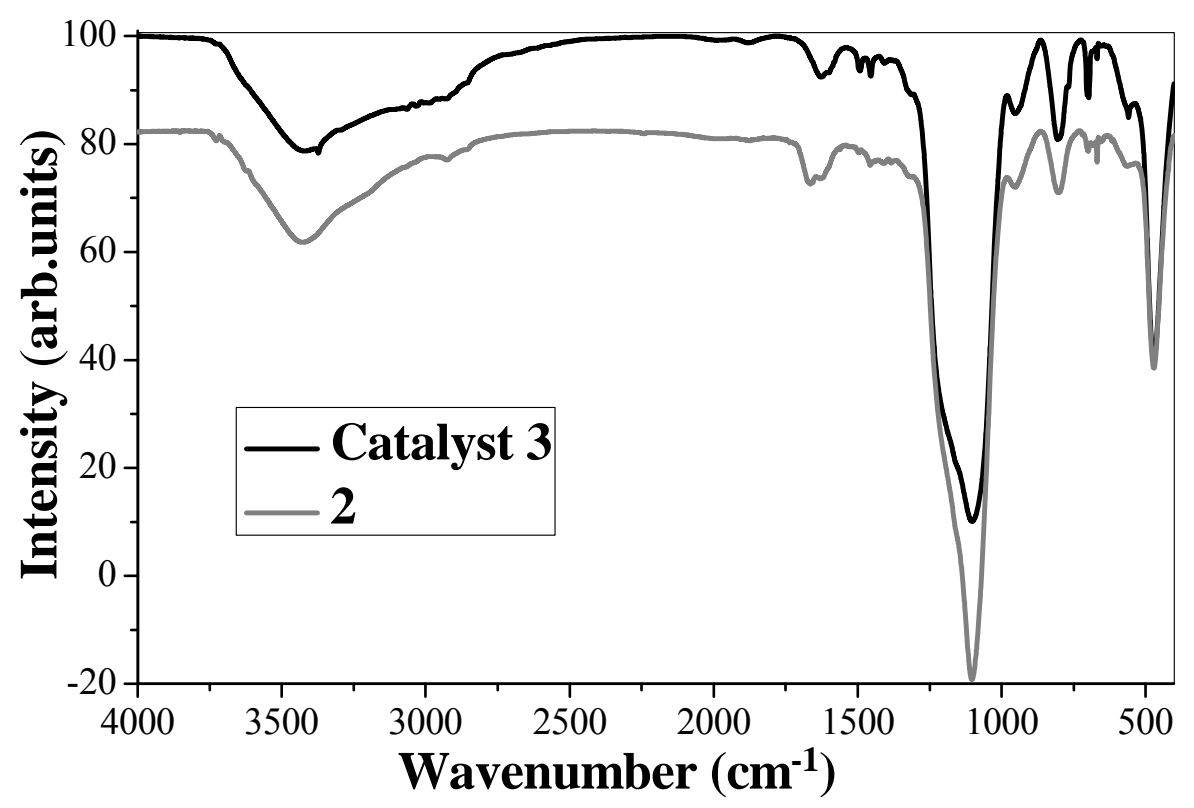

Figure S1. FT-IR spectra of 2 and catalyst 3.

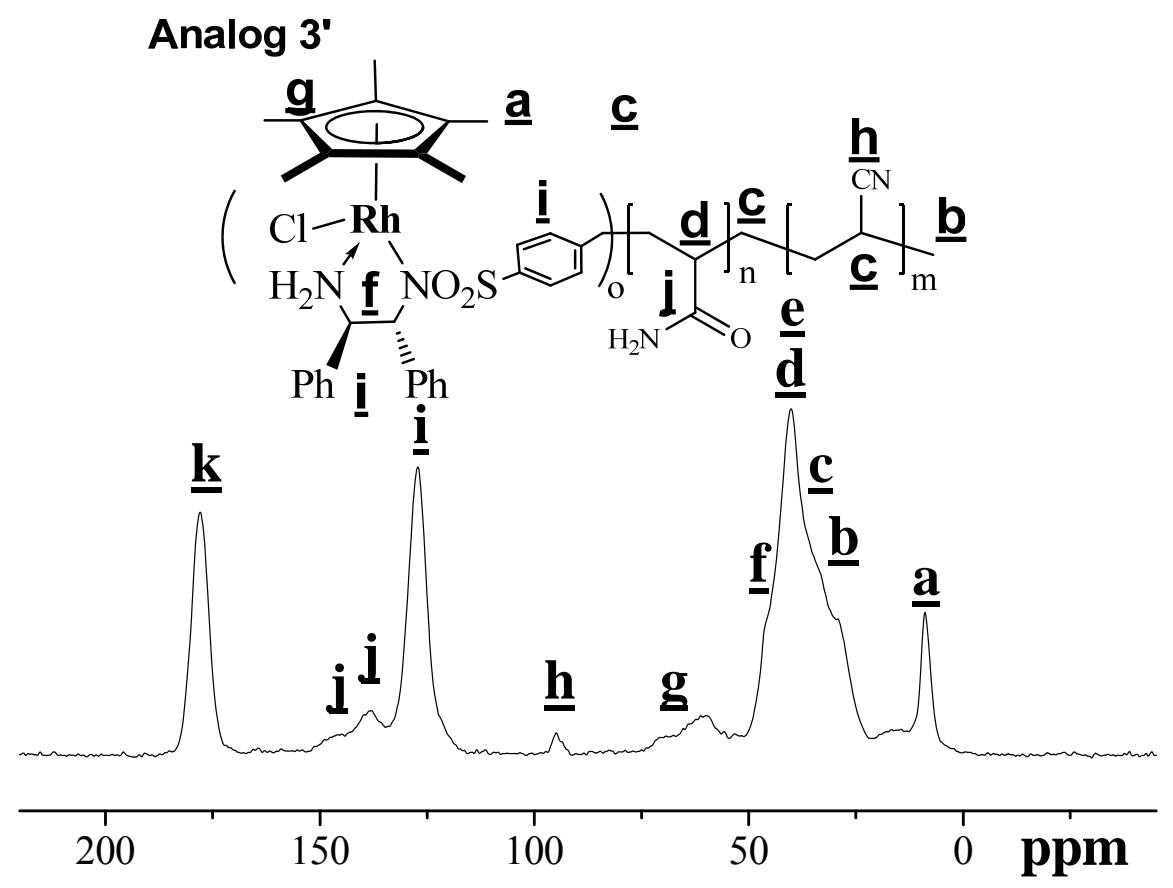

Figure S2. ${ }^{13} \mathrm{C}$ CP MAS NMR spectrum of its polymeric analog 3'. 


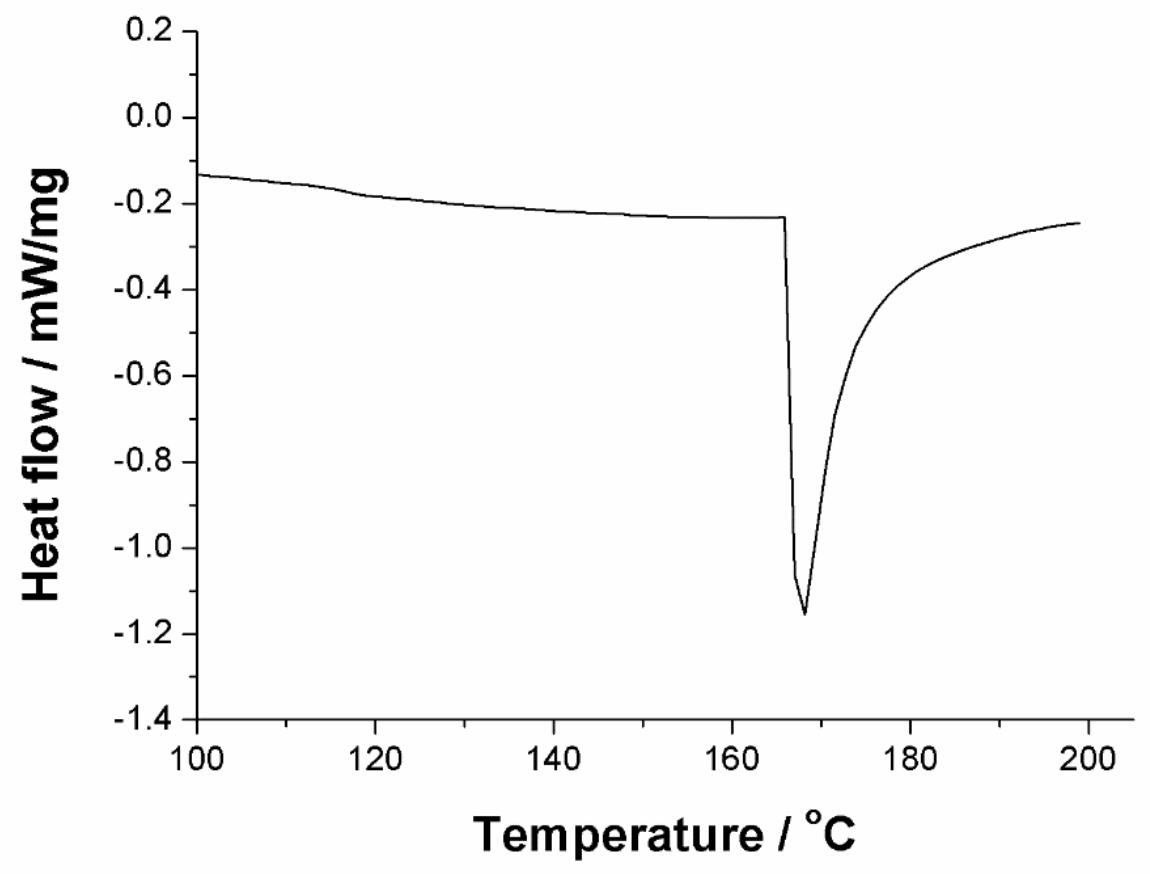

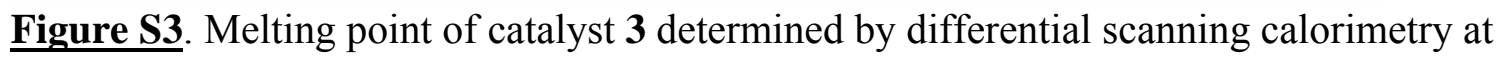
a heating rate of $30 \mathrm{~K} / \mathrm{min}$.

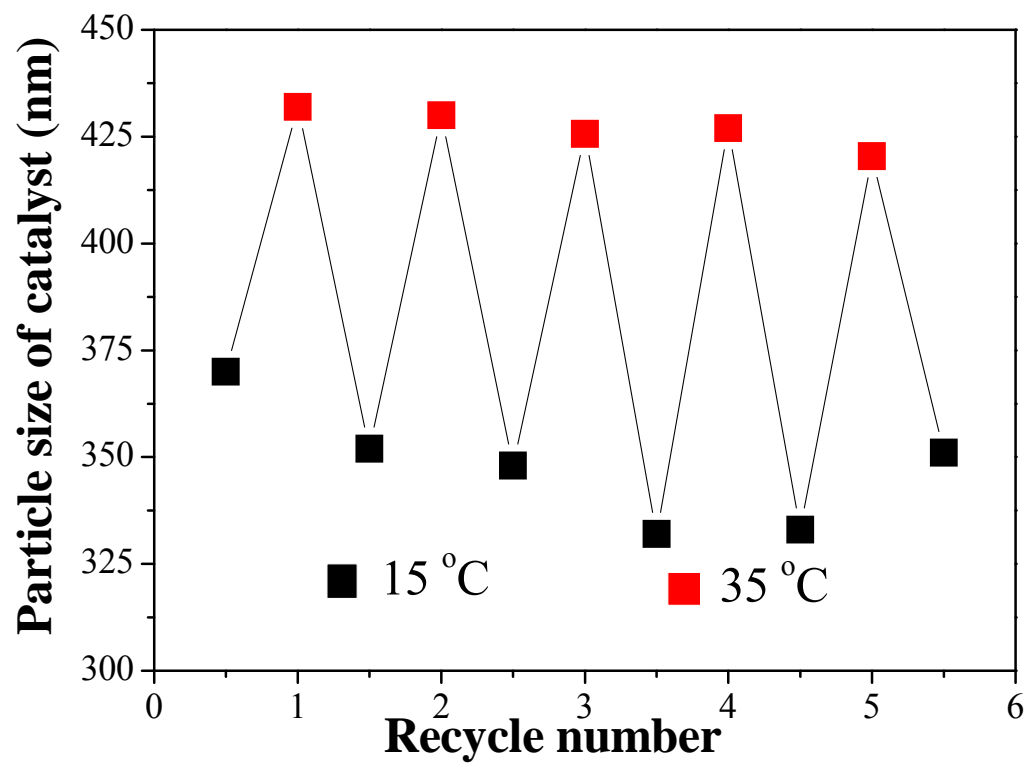

Figure S4. Average hydrodynamic diameters distribution measurement for the recycled catalyst 3 at indicated $15^{\circ} \mathrm{C}$ and $35^{\circ} \mathrm{C}$. 
Results $\left(15^{\circ} \mathrm{C}\right.$ for first rum)

$\begin{array}{rlllll} & & & \text { Diam. (nm) } & \text { \% Intensity } & \text { Width (nm) } \\ \text { Z-Average (d.nm): } & 377.0 & \text { Peak 1: } & 388.9 & 79.9 & 207.3 \\ \text { Pdl: } & 0.337 & \text { Peak 2: } & 2552 & 20.1 & 1181 \\ \text { Intercept: } & 0.953 & \text { Peak 3: } & 0.000 & 0.0 & 0.000 \\ \text { Result quality } & \text { Good } & & & & \end{array}$

Size Distribution by Intensity

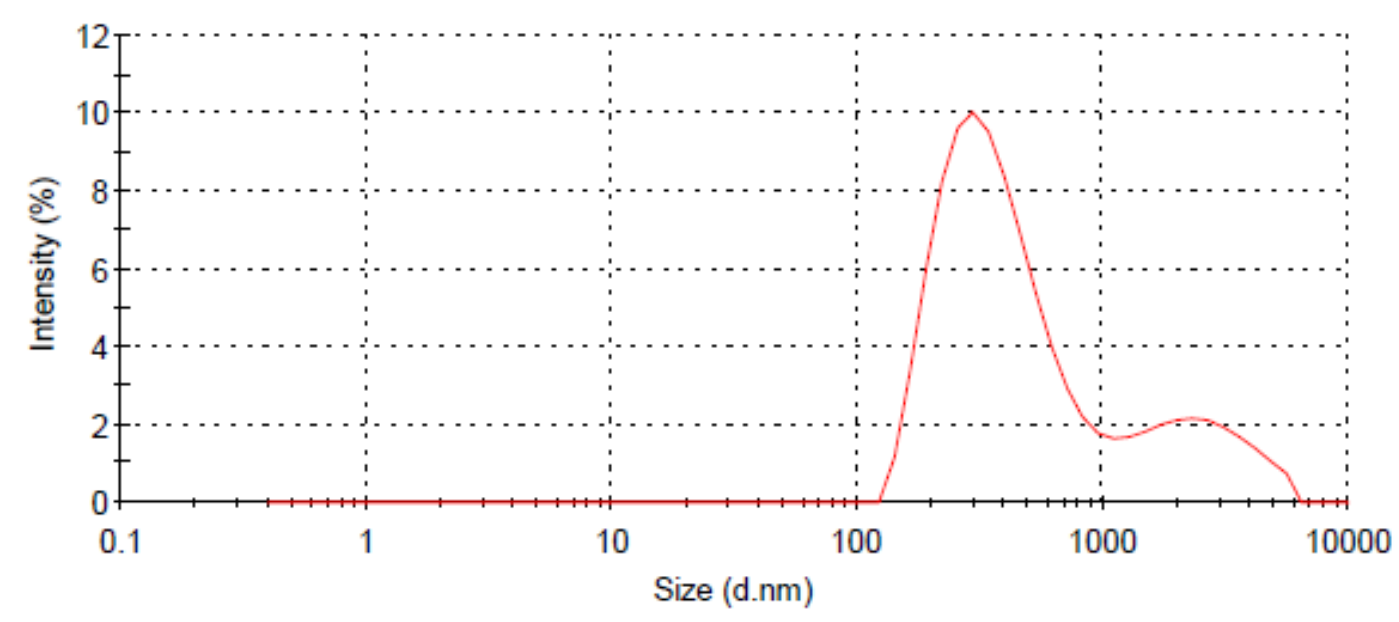

Results $\left(35^{\circ} \mathrm{C}\right.$ for first rum)

$\begin{array}{rlllll} & & \text { Diam. (nm) } & \text { \% Intensity } & \text { Width (nm) } \\ \text { Z-Average (d.nm): } & 432.4 & \text { Peak 1: } & 391.5 & 89.6 & 133.2 \\ \text { Pdl: } & 0.335 & \text { Peak 2: } & 4718 & 10.4 & 788.7 \\ \text { Intercept: } & 0.962 & \text { Peak 3: } & 0.000 & 0.0 & 0.000 \\ \text { Result quality } & \text { Good } & & & & \end{array}$

Size Distribution by Intensity

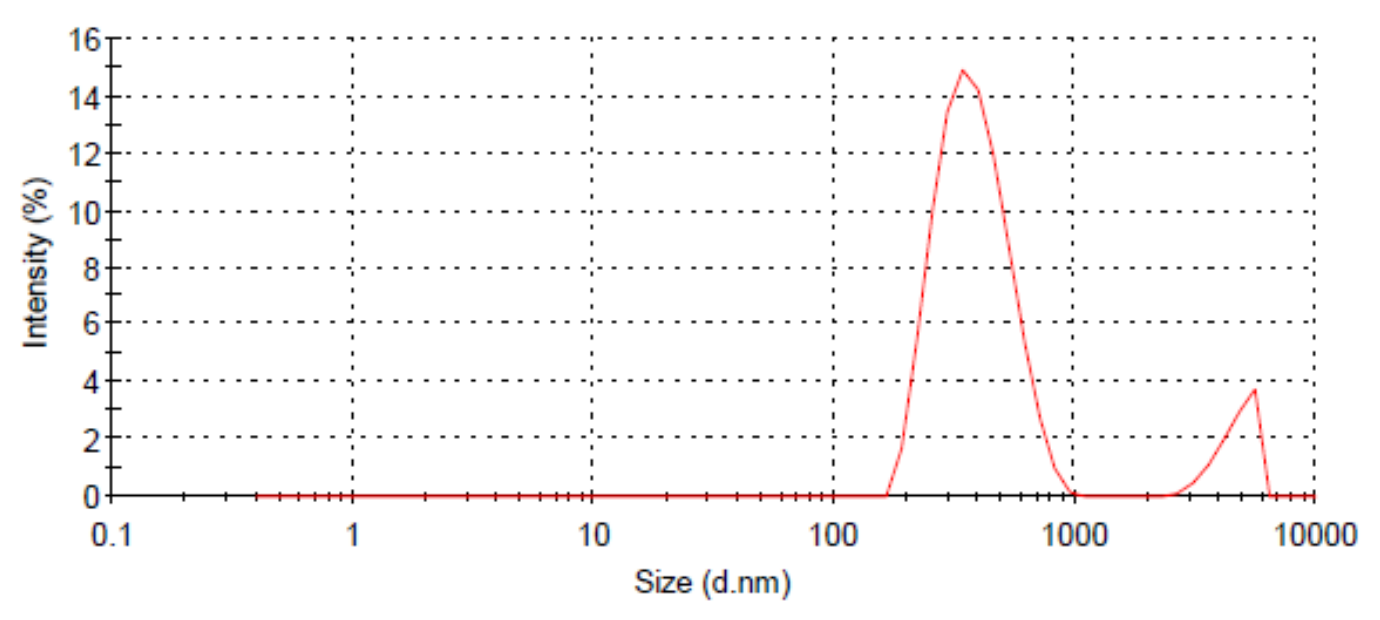


Results $\left(15^{\circ} \mathrm{C}\right.$ for second rum)

$\begin{array}{rlllll} & & & \text { Diam. (nm) } & \text { \% Intensity } & \text { Width (nm) } \\ \text { Z-Average (d.nm): } & 352.7 & \text { Peak 1: } & 413.1 & 95.8 & 211.2 \\ \text { Pdl: } & 0.277 & \text { Peak 2: } & 4782 & 4.2 & 733.7 \\ \text { Intercept: } 0.952 & \text { Peak 3: } & 0.000 & 0.0 & 0.000 \\ \text { Result quality } & \text { Good } & & & & \end{array}$

Size Distribution by Intensity



\section{Results $\left(35^{\circ} \mathrm{C}\right.$ for second rum)}

\begin{tabular}{|c|c|c|c|c|}
\hline 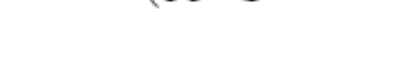 & & Diam. (nm) & $\%$ Intensity & Width $(\mathrm{nm})$ \\
\hline Z-Average (d.nm): 430.9 & Peak 1: & 437.8 & 92.0 & 183.6 \\
\hline Pdl: 0.280 & Peak 2: & 4623 & 8.0 & 822.8 \\
\hline Intercept: 0.953 & Peak 3: & 0.000 & 0.0 & 0.000 \\
\hline Result quality Good & & & & \\
\hline
\end{tabular}

Size Distribution by Intensity

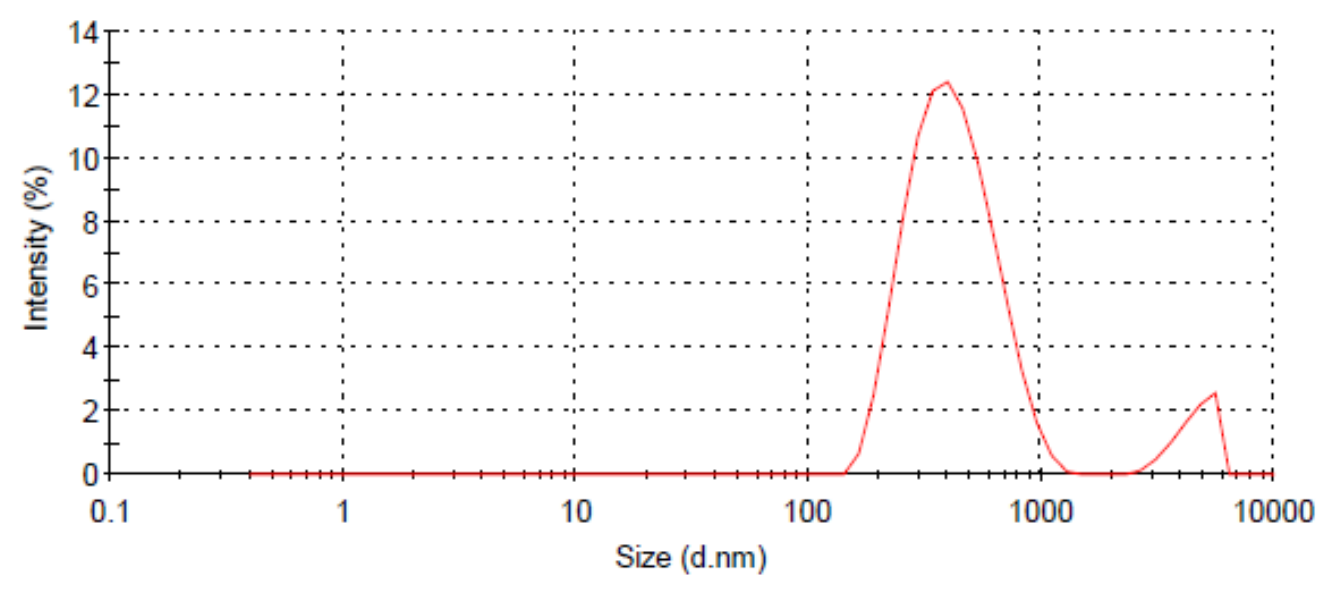


Results $\left(15^{\circ} \mathrm{C}\right.$ for third rum)

$\begin{array}{rlllll} & & \text { Diam. }(\mathrm{nm}) & \% \text { Intensity } & \text { Width (nm) } \\ \text { Z-Average (d.nm): } & 348.3 & \text { Peak 1: } & 534.4 & 100.0 & 401.9 \\ \text { Pdl: } & 0.267 & \text { Peak 2: } & 0.000 & 0.0 & 0.000 \\ \text { Intercept: } & 0.959 & \text { Peak 3: } & 0.000 & 0.0 & 0.000 \\ \text { Result quality } & \text { Good } & & & & \end{array}$

Size Distribution by Intensity

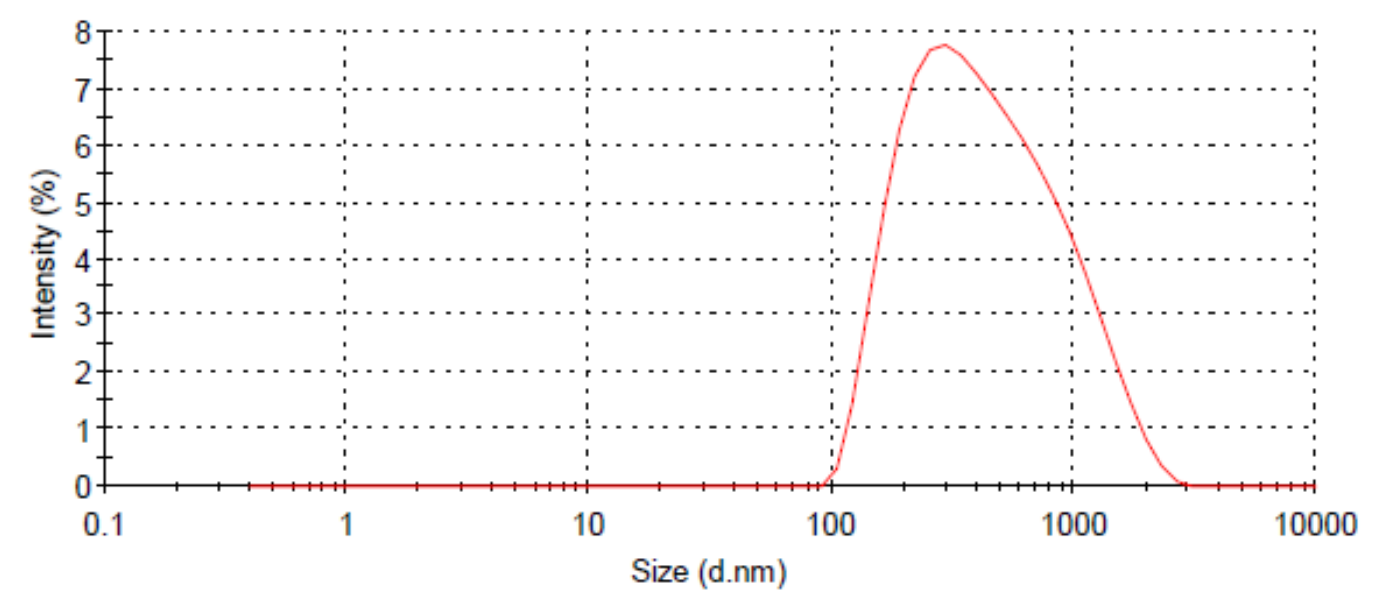

\section{Results $\left(35^{\circ} \mathrm{C}\right.$ for third rum)}

$\begin{array}{rlllll} & & \text { Diam. (nm) } & \text { \% Intensity } & \text { Width (nm) } \\ \text { Z-Average (d.nm): } & 425.7 & \text { Peak 1: } & 412.8 & 98.0 & 128.9 \\ \text { Pdl: } & 0.281 & \text { Peak 2: } & 5407 & 2.0 & 304.0 \\ \text { Intercept: } & 0.958 & \text { Peak 3: } & 0.000 & 0.0 & 0.000 \\ \text { Result quality } & \text { Good } & & & & \end{array}$

Size Distribution by Intensity

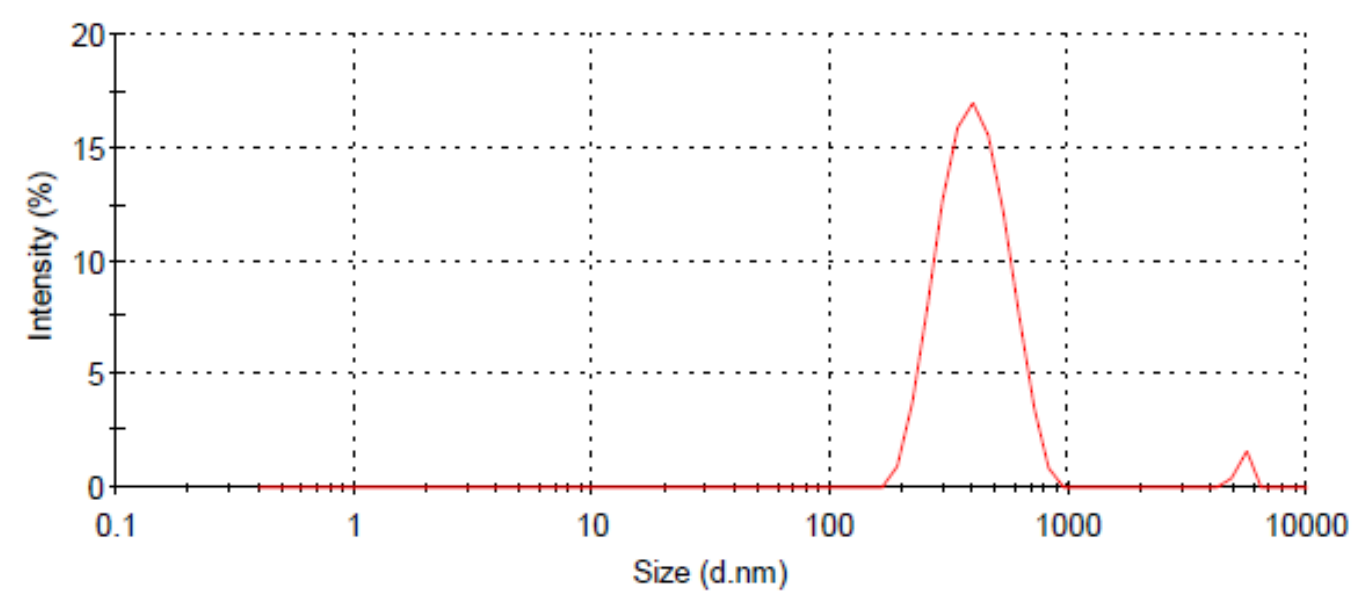




$\begin{array}{rlllll}\text { Results }\left(\mathbf{1 5}{ }^{\mathbf{0}} \mathbf{C} \text { for fourth rum) }\right. & & \text { Diam. (nm) } & \text { \% Intensity } & \text { Width (nm) } \\ \text { Z-Average (d.nm): } & 332.3 & \text { Peak 1: } & 351.7 & 95.5 & 126.5 \\ \text { Pdl: } & 0.281 & \text { Peak 2: } & 4997 & 4.5 & 610.0 \\ \text { Intercept: } & 0.946 & \text { Peak 3: } & 0.000 & 0.0 & 0.000 \\ \text { Result quality } & \text { Good } & & & & \end{array}$

Size Distribution by Intensity

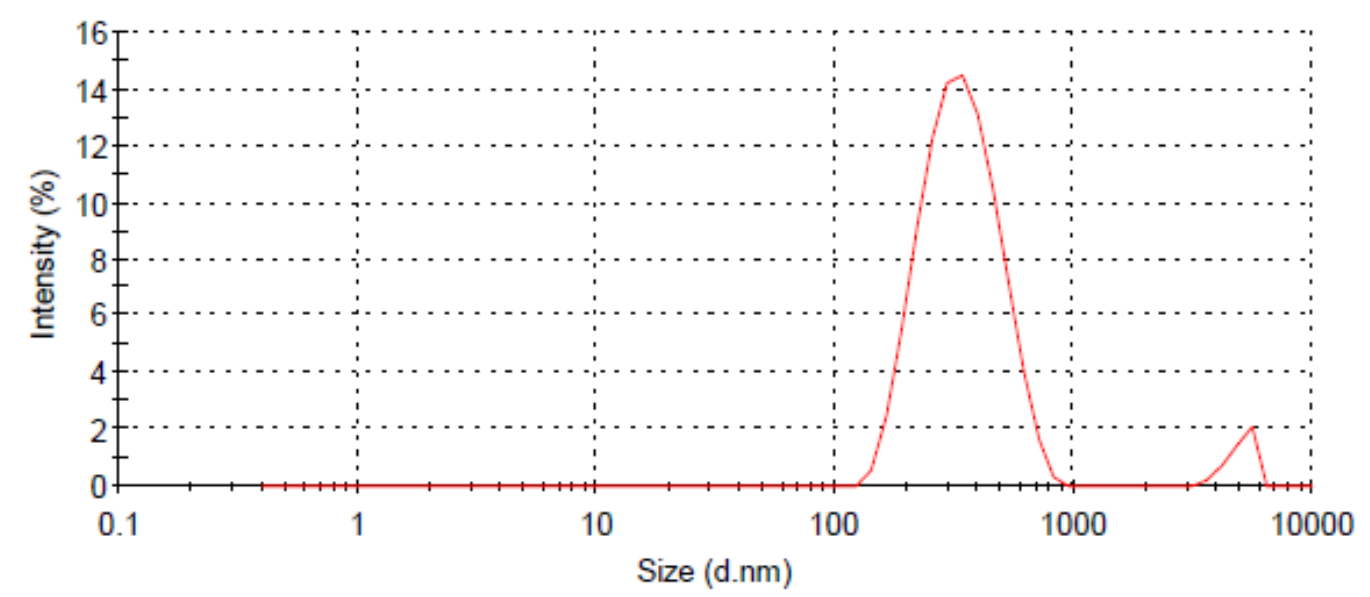

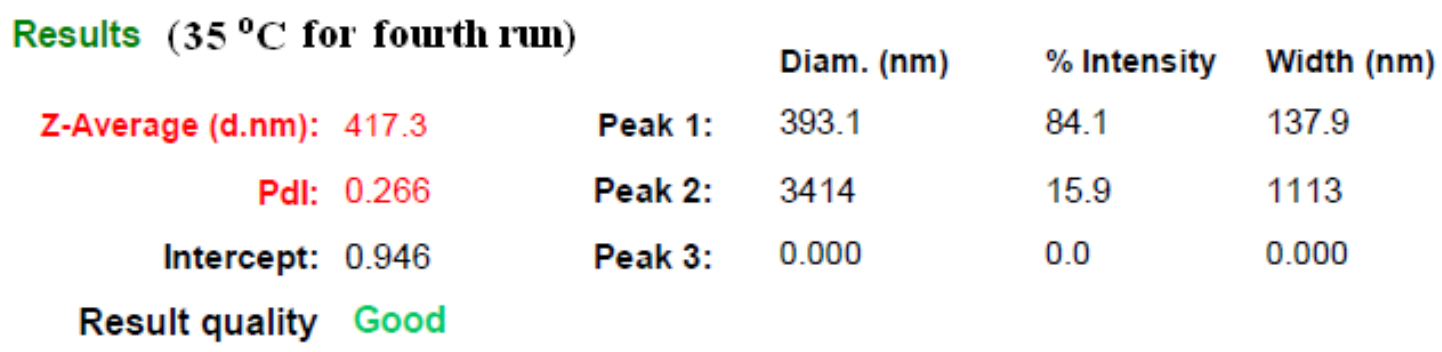

Size Distribution by Intensity

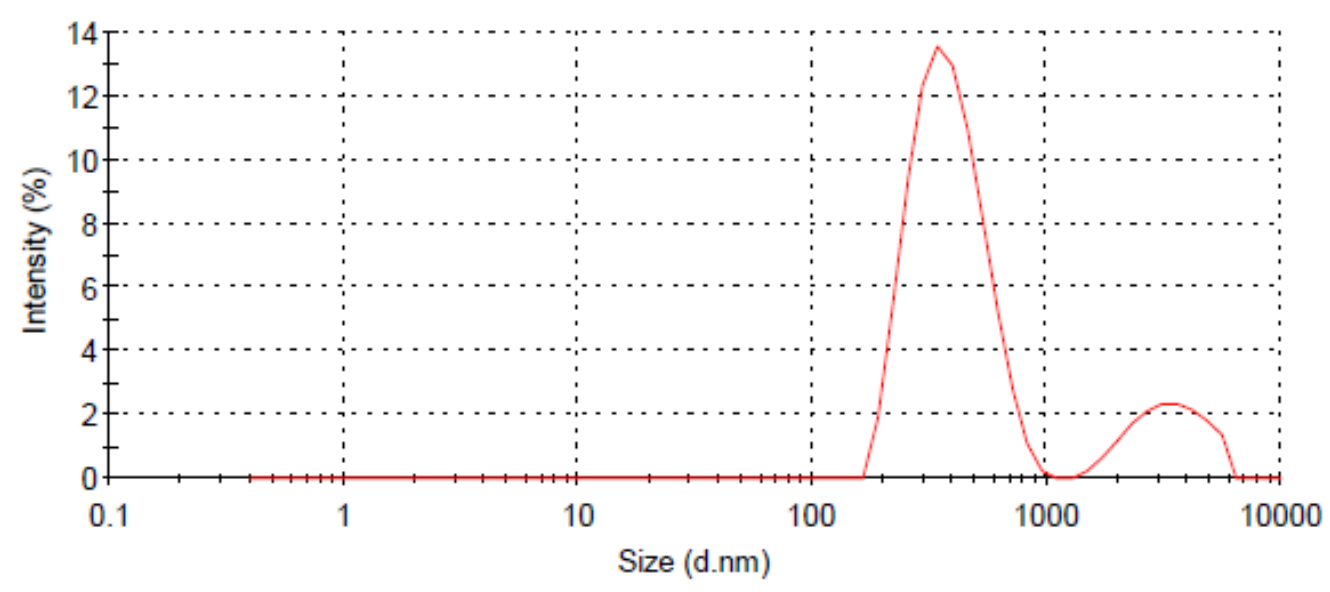


Results $\left(15^{\circ} \mathrm{C}\right.$ for fifth rum)

$\begin{array}{llll} & \text { Diam. }(\mathbf{n m}) & \% \text { Intensity } & \text { Width }(\mathbf{n m}) \\ \text { Peak 1: } & 325.4 & 90.2 & 111.1 \\ \text { Peak 2: } & 4192 & 9.8 & 998.0 \\ \text { Peak 3: } & 0.000 & 0.0 & 0.000\end{array}$

Size Distribution by Intensity

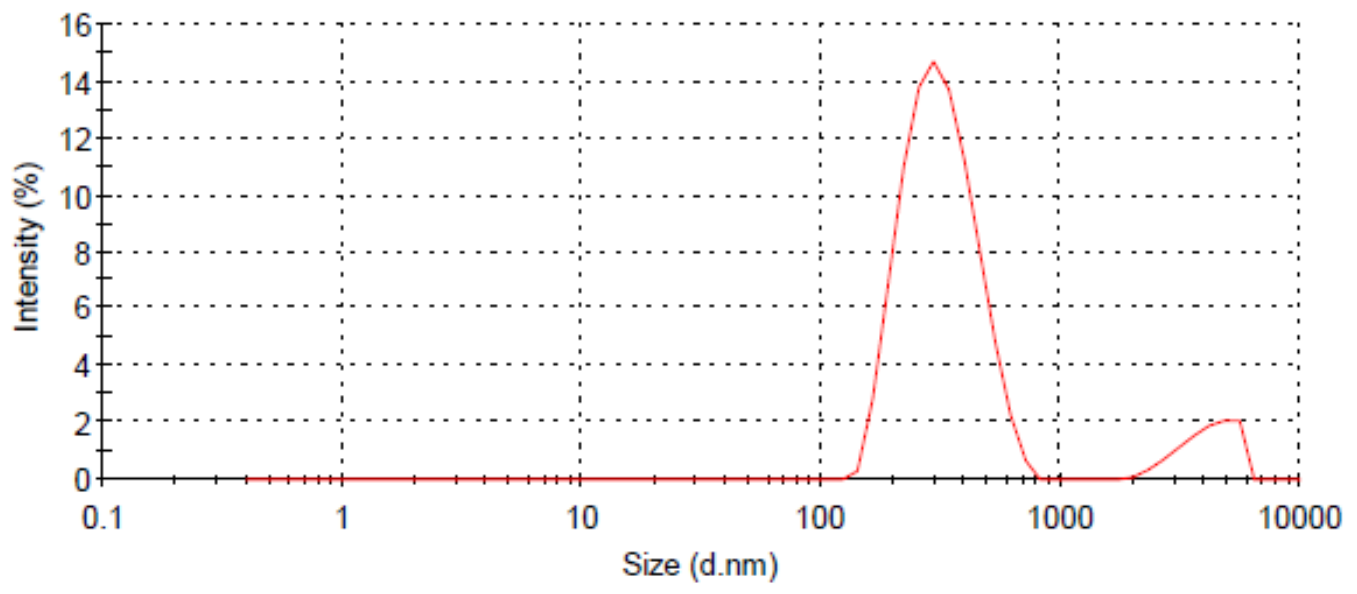

Results $\left(35^{\circ} \mathrm{C}\right.$ for fifth run)

$\begin{array}{rlllll} & & \text { Diam. (nm) } & \text { \% Intensity } & \text { Width (nm) } \\ \text { Z-Average (d.nm): } & 420.4 & \text { Peak 1: } & 410.6 & 96.5 & 136.2 \\ \text { Pdl: } & 0.281 & \text { Peak 2: } & 5225 & 3.5 & 460.1 \\ \text { Intercept: } & 0.962 & \text { Peak 3: } & 0.000 & 0.0 & 0.000 \\ \text { Result quality } & \text { Good } & & & & \end{array}$

Size Distribution by Intensity

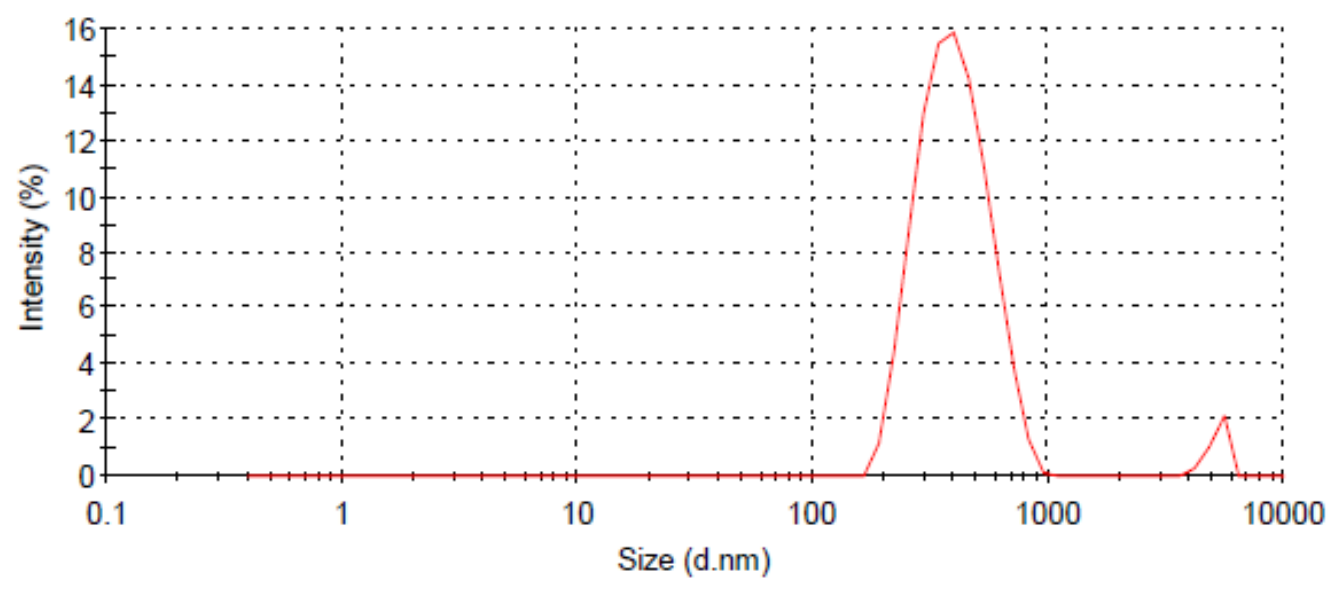


5a (entry 1 in Table 1): (S)-3-benzylisobenzofuran-1(3H)-one [Org. Lett., 2009, 11,

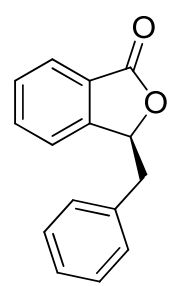

4712]. Yield: 99\% (98\% ee); ${ }^{1} \mathrm{H}$ NMR (400 $\left.\mathrm{MHz} \mathrm{CDCl}_{3}\right): \delta 3.13-$ $3.18(\mathrm{dd}, J=6 \mathrm{~Hz}, J=14 \mathrm{~Hz}, 1 \mathrm{H}), 3.22-3.27(\mathrm{dd}, J=6.8 \mathrm{~Hz}, J=$ $14 \mathrm{~Hz}, 1 \mathrm{H}), 5.67$ (t, $J=6.4 \mathrm{~Hz}, 1 \mathrm{H}), 7.18-7.29(\mathrm{~m}, 6 \mathrm{H}), 7.46(\mathrm{t}, J=$ $7.5 \mathrm{~Hz}, 1 \mathrm{H}), 7.59(\mathrm{dt}, J=0.3, J=7.5 \mathrm{~Hz}, 1 \mathrm{H}), 7.82(\mathrm{~d}, J=7.6 \mathrm{~Hz}$, 5a (entry 1 in Table 1) $1 \mathrm{H}) ;{ }^{13} \mathrm{C}\left\{{ }^{1} \mathrm{H}\right\}$ NMR (101 MHz, $\left.\mathrm{CDCl}_{3}\right): \delta 40.83,81.27,122.40$, $125.62,126.22,127.16,129.01,129.23,129.74,133.82,135.07,149.16,170.31 ; \mathrm{GC} / \mathrm{MS}$ $(\mathrm{m} / \mathrm{z}): 224$.

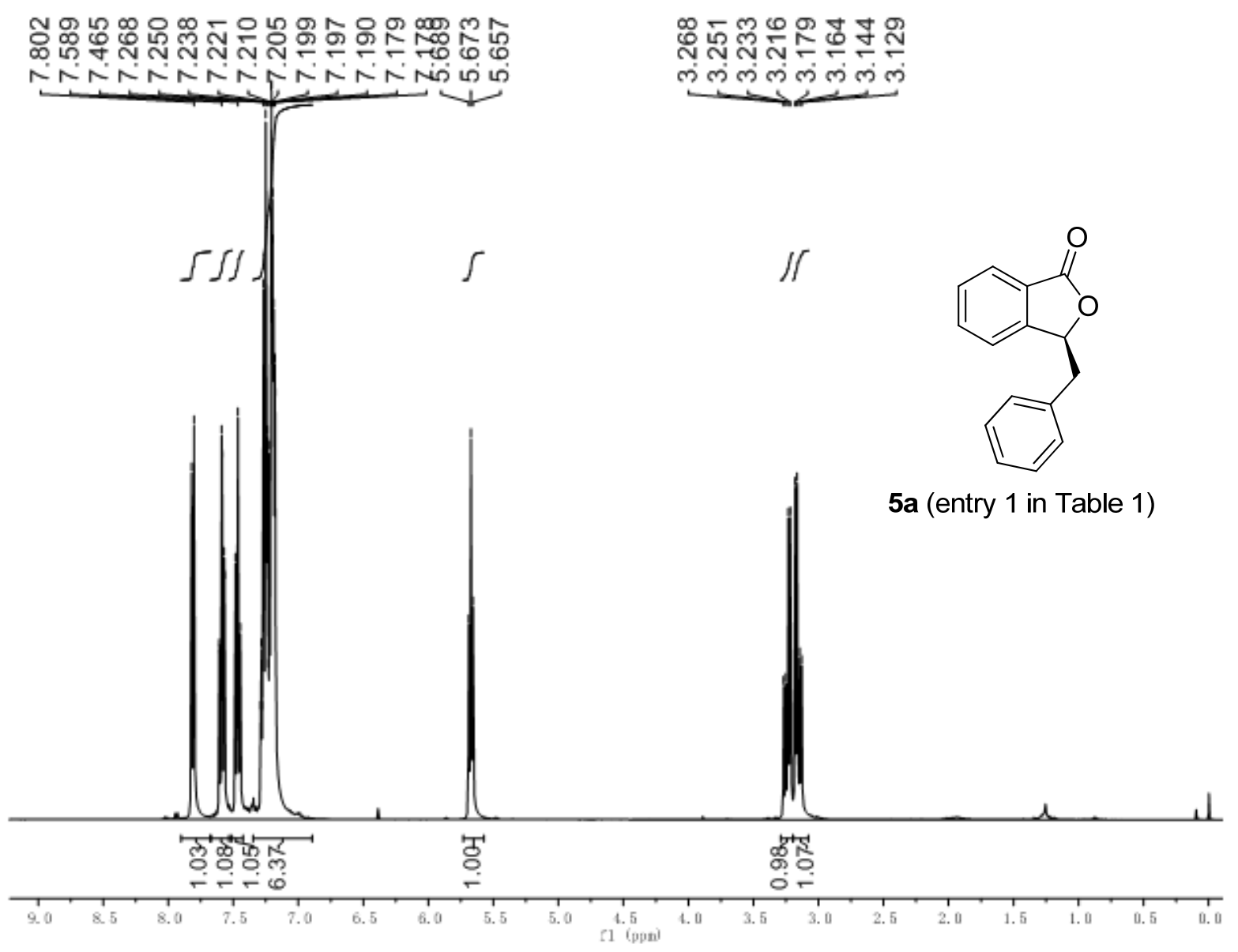



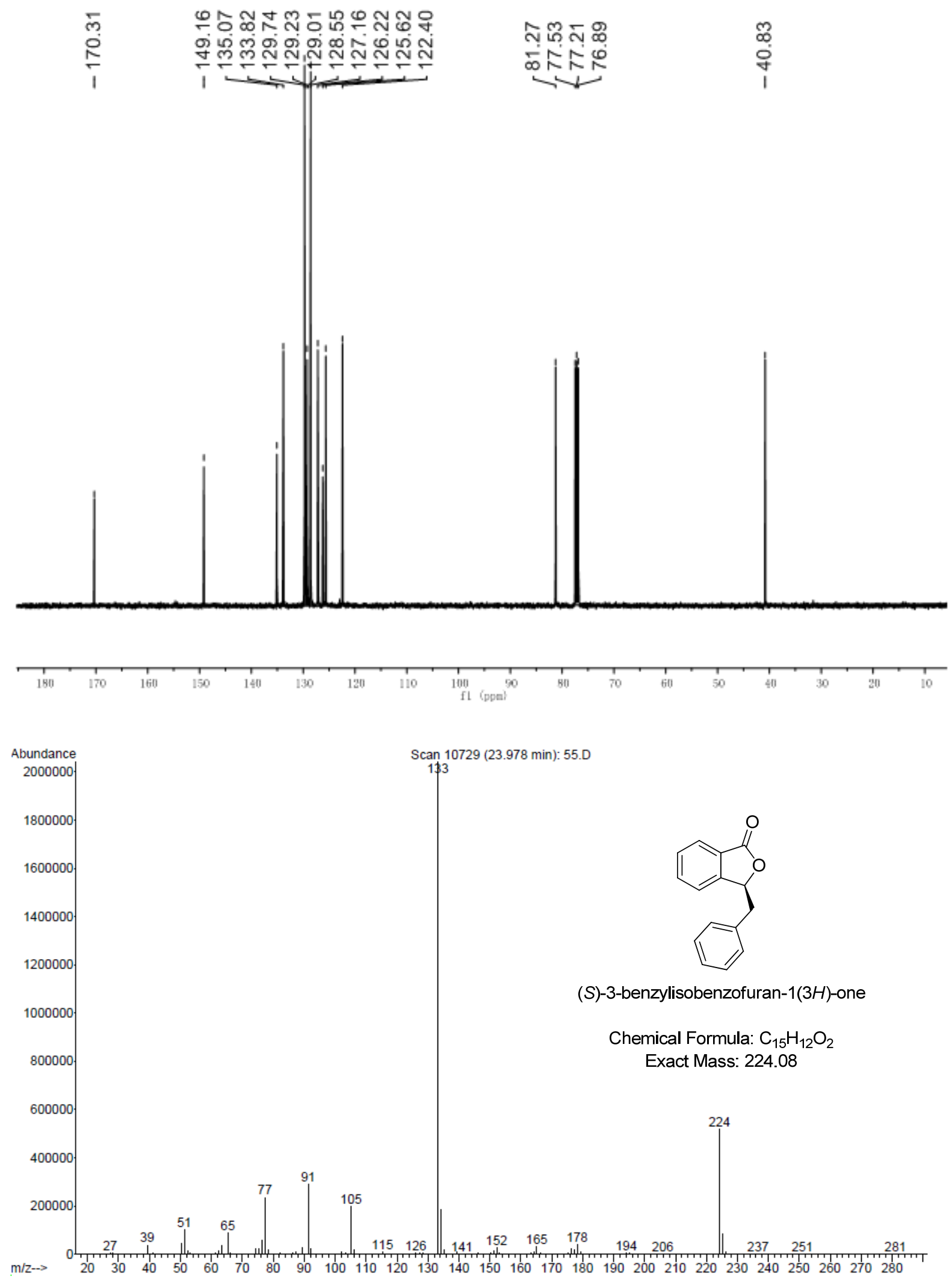
5b (entry 6 in Table 1): (S)-3-(4-fluorobenzyl)isobenzofuran-1(3H)-one [Org. Lett., 2009,

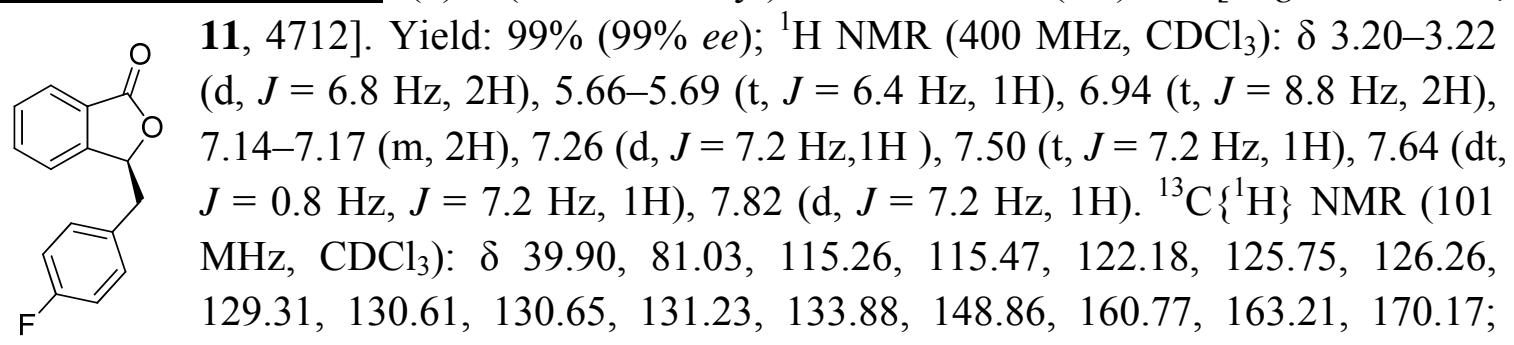
GC/MS (m/z): 242.

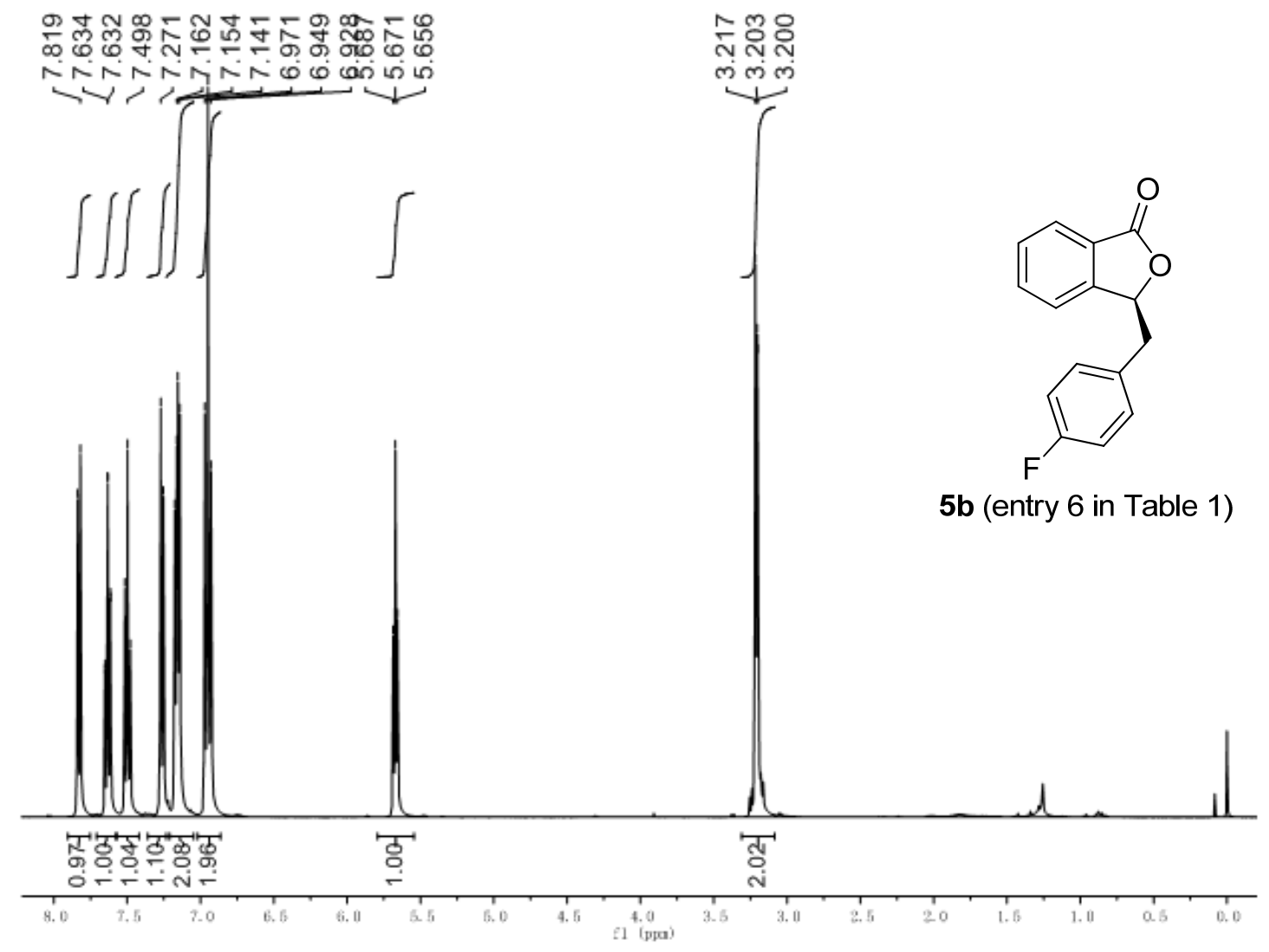




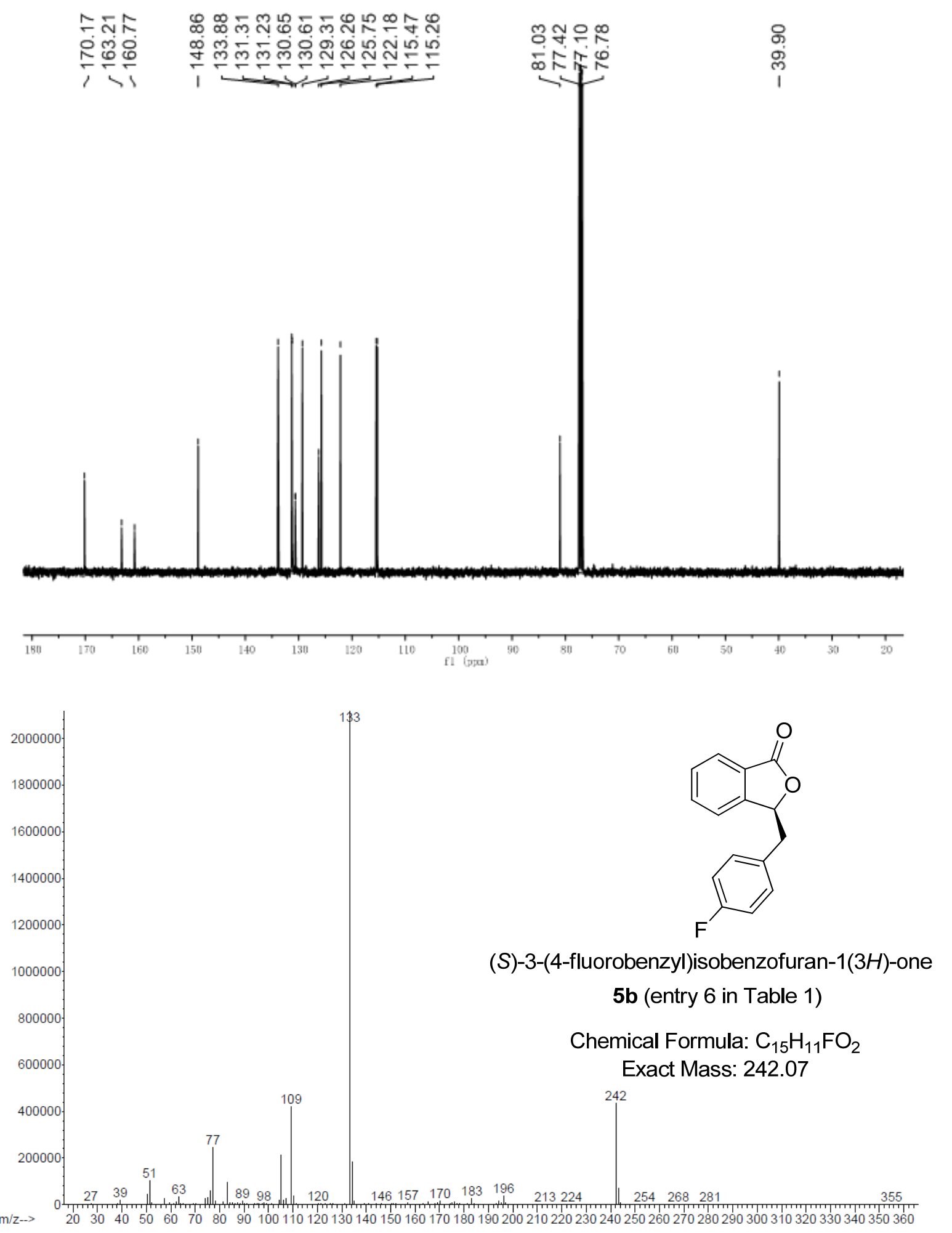


5c (entry 7 in Table 1): (S)-3-(4-chlorobenzyl)isobenzofuran-1(3H)-one [Org. Lett., 2009, 11, 4712]. Yield : 99\% (99\% ee); ${ }^{1} \mathrm{H} \mathrm{NMR}\left(400 \mathrm{MHz}, \mathrm{CDCl}_{3}\right): \delta 3.20-3.22$
$(\mathrm{~d}, J=6 \mathrm{~Hz}, 2 \mathrm{H}), 5.66-5.69(\mathrm{t}, J=6.4 \mathrm{~Hz}, 1 \mathrm{H}), 7.13(\mathrm{~d}, J=8.4 \mathrm{~Hz}, 2 \mathrm{H})$,
$\begin{aligned} & 7.22-7.27(\mathrm{~m}, 3 \mathrm{H}), 7.50(\mathrm{t}, J=7.2 \mathrm{~Hz}, 1 \mathrm{H}), 7.64(\mathrm{dt}, J=0.8 \mathrm{~Hz}, J=7.2 \mathrm{~Hz}, \\ & 1 \mathrm{H}), 7.84(\mathrm{~d}, J=7.2 \mathrm{~Hz}, 1 \mathrm{H}) .{ }^{13} \mathrm{C}\left\{{ }^{1} \mathrm{H}\right\} \mathrm{NMR}\left(101 \mathrm{MHz}, \mathrm{CDCl}_{3}\right): \delta 40.08, \\ & 80.79,122.12,125.84,126.27,128.67,129.36,131.07,133.11,133.39, \\ & 133.88,148.75,170.07 ; \mathrm{GC} / \mathrm{MS}(\mathrm{m} / \mathrm{z}): 258 .\end{aligned}$

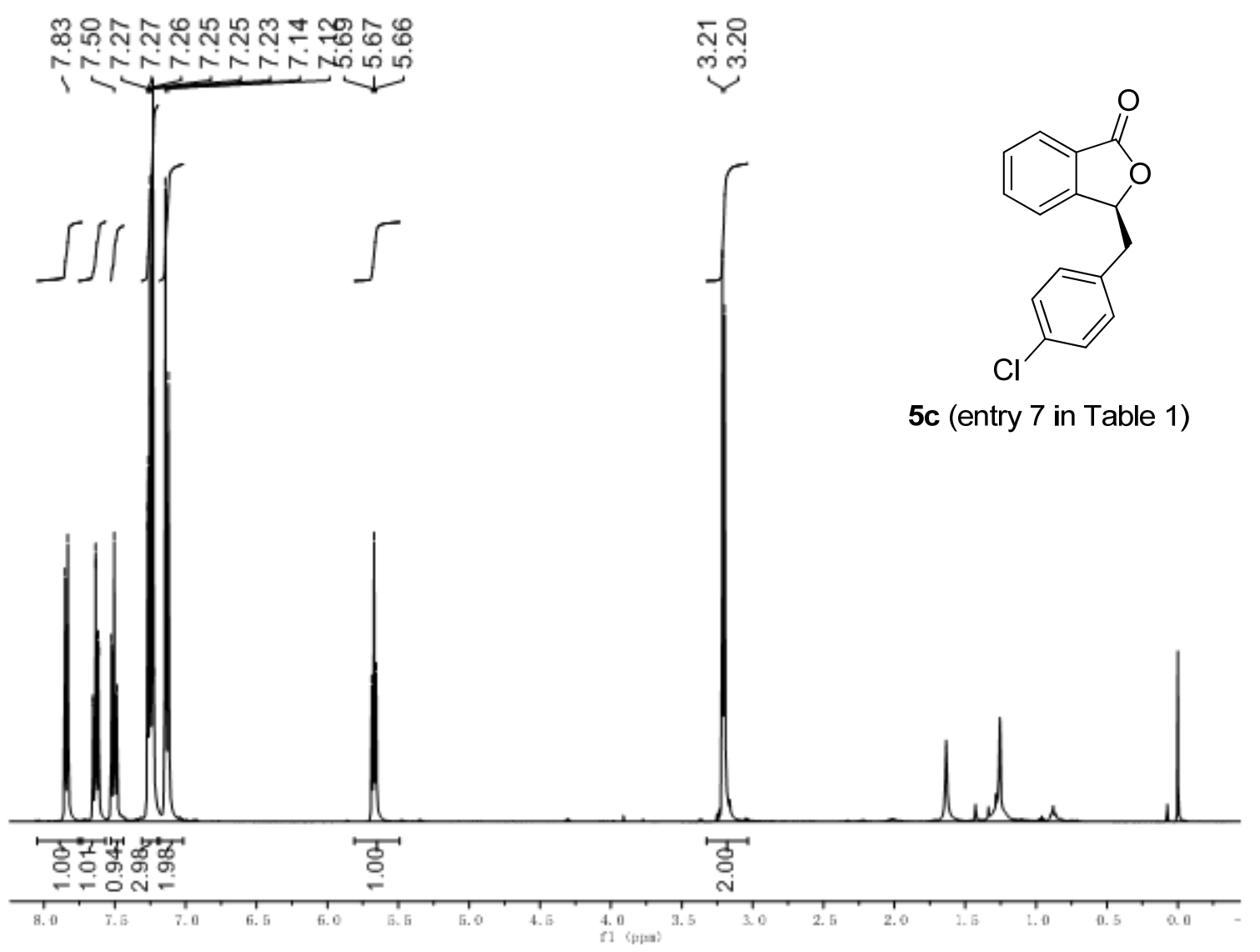




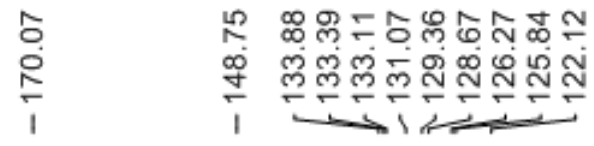

윰유요

敋华

$\infty$
$\stackrel{0}{0}$
+
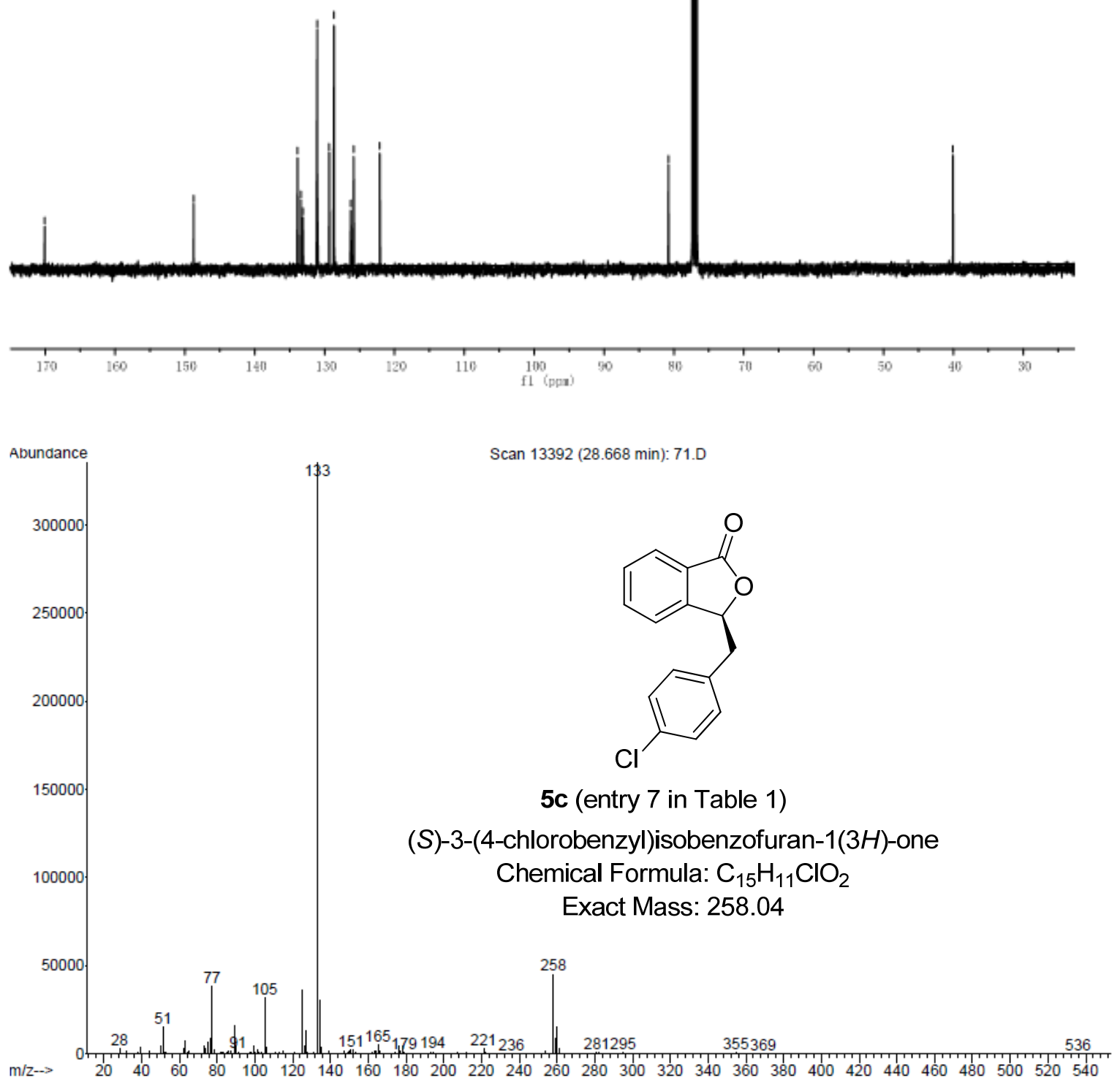
5d (entry 8 in Table 1): (S)-3-(2-chlorobenzyl)isobenzofuran-1(3H)-one [Org. Lett., 2009, 11, 4712]. Yield : 98\% (98\% ee); ${ }^{1} \mathrm{H}$ NMR (400 MHz, $\left.\mathrm{CDCl}_{3}\right): \delta 3.22-3.25$ (dd, $J=8 \mathrm{~Hz}, J=14 \mathrm{~Hz}, 1 \mathrm{H}), 3.39-3.43(\mathrm{dd}, J=8 \mathrm{~Hz}, J=14 \mathrm{~Hz}, 1 \mathrm{H})$, 5.76-5.79 (t, $J=6.4 \mathrm{~Hz}, 1 \mathrm{H}), 7.23-7.34(\mathrm{~m}, 4 \mathrm{H}), 7.39-7.42(\mathrm{~m}, 1 \mathrm{H}), 7.53(\mathrm{t}$, $J=7.5 \mathrm{~Hz}, 1 \mathrm{H}), 7.65$ (dt, $J=1 \mathrm{~Hz}, J=7.5 \mathrm{~Hz}, 1 \mathrm{H}), 7.89(\mathrm{~d}, J=7.6 \mathrm{~Hz}, 1 \mathrm{H})$. ${ }^{13} \mathrm{C}\left\{{ }^{1} \mathrm{H}\right\}$ NMR $\left(101 \mathrm{MHz}, \mathrm{CDCl}_{3}\right): \delta 39.00,79.87,122.38,125.77,126.12$, $127.06,128.85,129.37,129.68,132.17,133.50,133.90,134.18,149.14$, 170.21; GC/MS (m/z): 258.

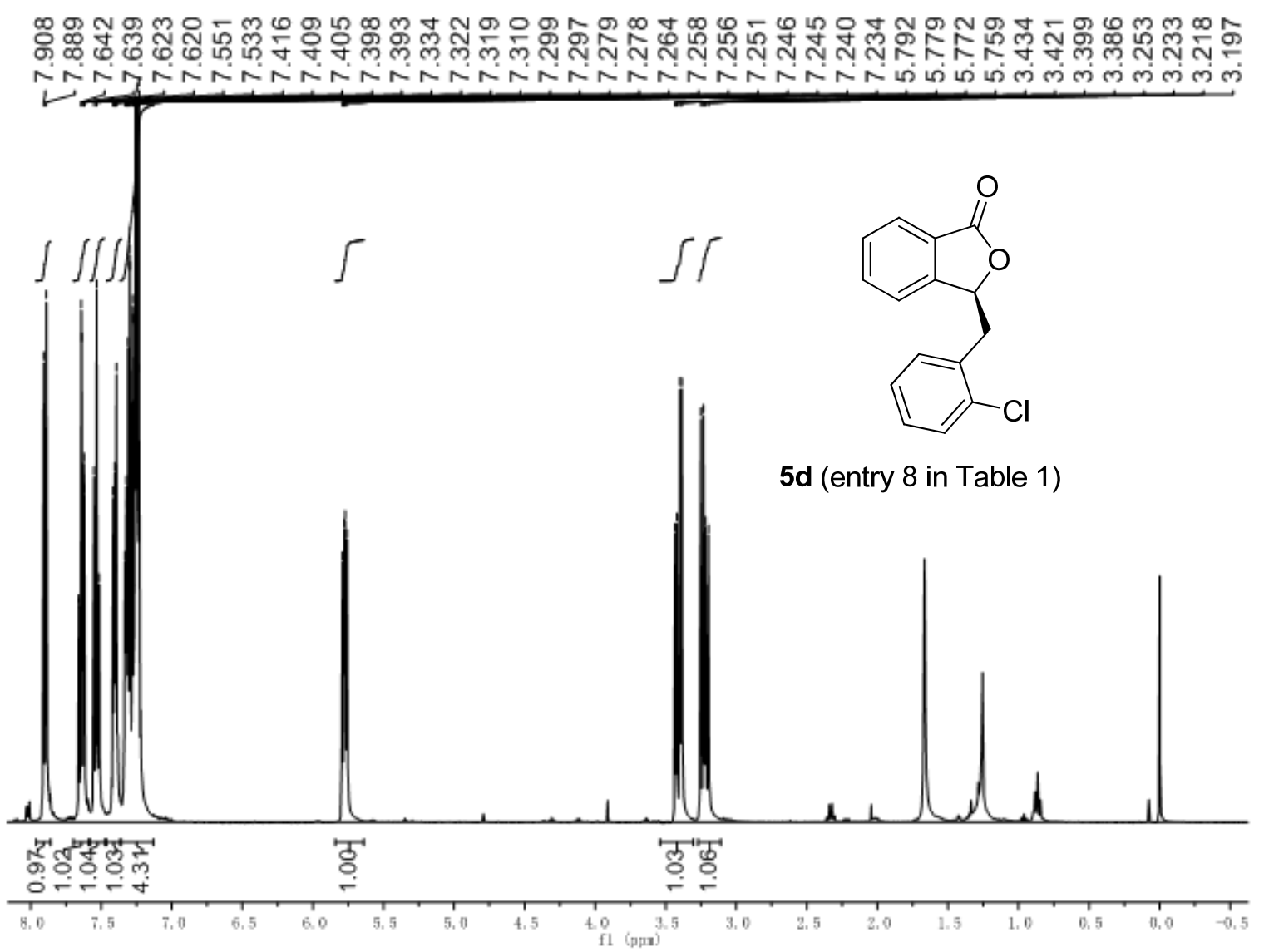




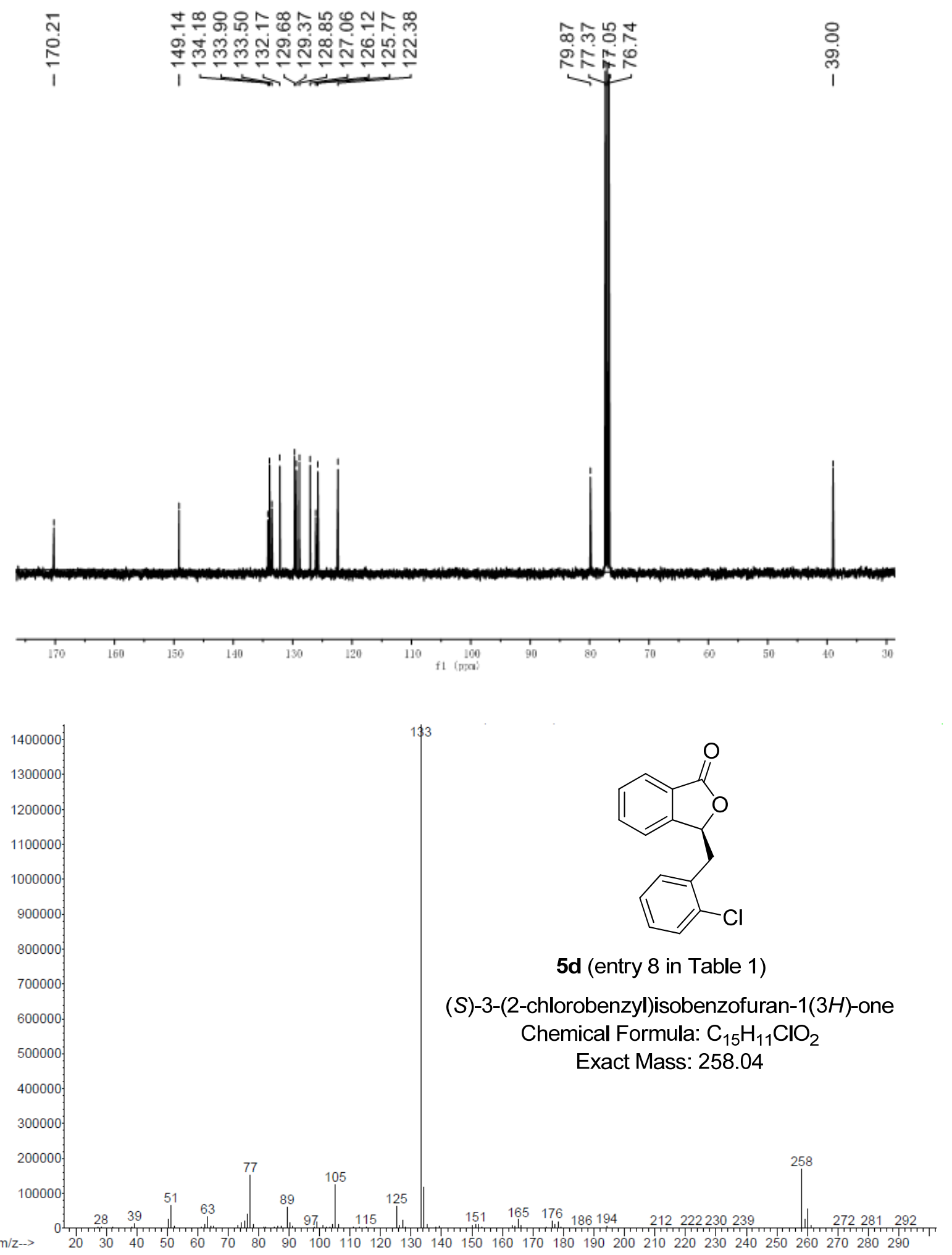


5e (entry 9 in Table 1): (S)-3-(4-(trifluoromethyl)benzyl)isobenzofuran-1(3H)-one [Org. Lett., 2009, 11, 4712]. Yield : 99\% (98\% ee); ${ }^{1} \mathrm{H}$ NMR $(400 \mathrm{MHz}$,<smiles>O=C1OC(Cc2ccc(C(F)(F)F)cc2)c2ccccc21</smiles>

$\left.\mathrm{CDCl}_{3}\right): \delta 3.21-3.27(\mathrm{dd}, J=6.8 \mathrm{~Hz}, J=14 \mathrm{~Hz} 1 \mathrm{H}), 3.32-3.37(\mathrm{dd}, J=$ $6.8 \mathrm{~Hz}, J=14 \mathrm{~Hz}, 1 \mathrm{H}), 5.70-5.73(\mathrm{t}, J=6 \mathrm{~Hz}, 1 \mathrm{H}), 7.33(\mathrm{t}, J=8 \mathrm{~Hz}$, $3 \mathrm{H}), 7.52(\mathrm{t} J=8.4 \mathrm{~Hz}, 3 \mathrm{H}), 7.67(\mathrm{dt}, J=0.8 \mathrm{~Hz}, J=7.6 \mathrm{~Hz}, 1 \mathrm{H}), 7.81(\mathrm{~d}$, $J=7.6 \mathrm{~Hz}, 1 \mathrm{H}) .{ }^{13} \mathrm{C}\left\{{ }^{1} \mathrm{H}\right\} \mathrm{NMR}\left(101 \mathrm{MHz}, \mathrm{CDCl}_{3}\right): \delta$ 40.21, $80.58,122.04,125.41,125.45,125,89,126.20,129.31,129.49,129.63$, 130.07, 134.03, 139.17, 148.62, 169.98; GC/MS (m/z): 292.

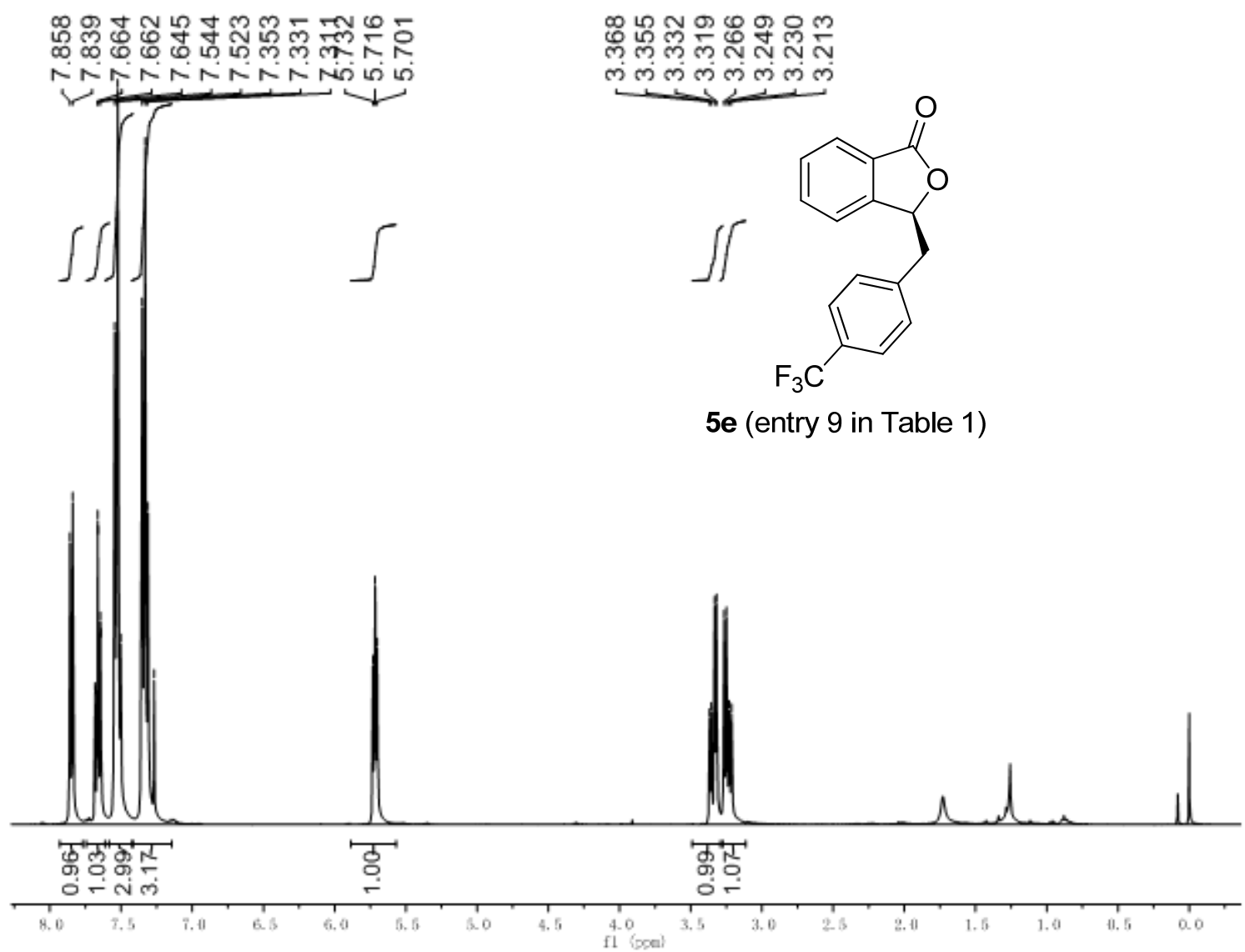



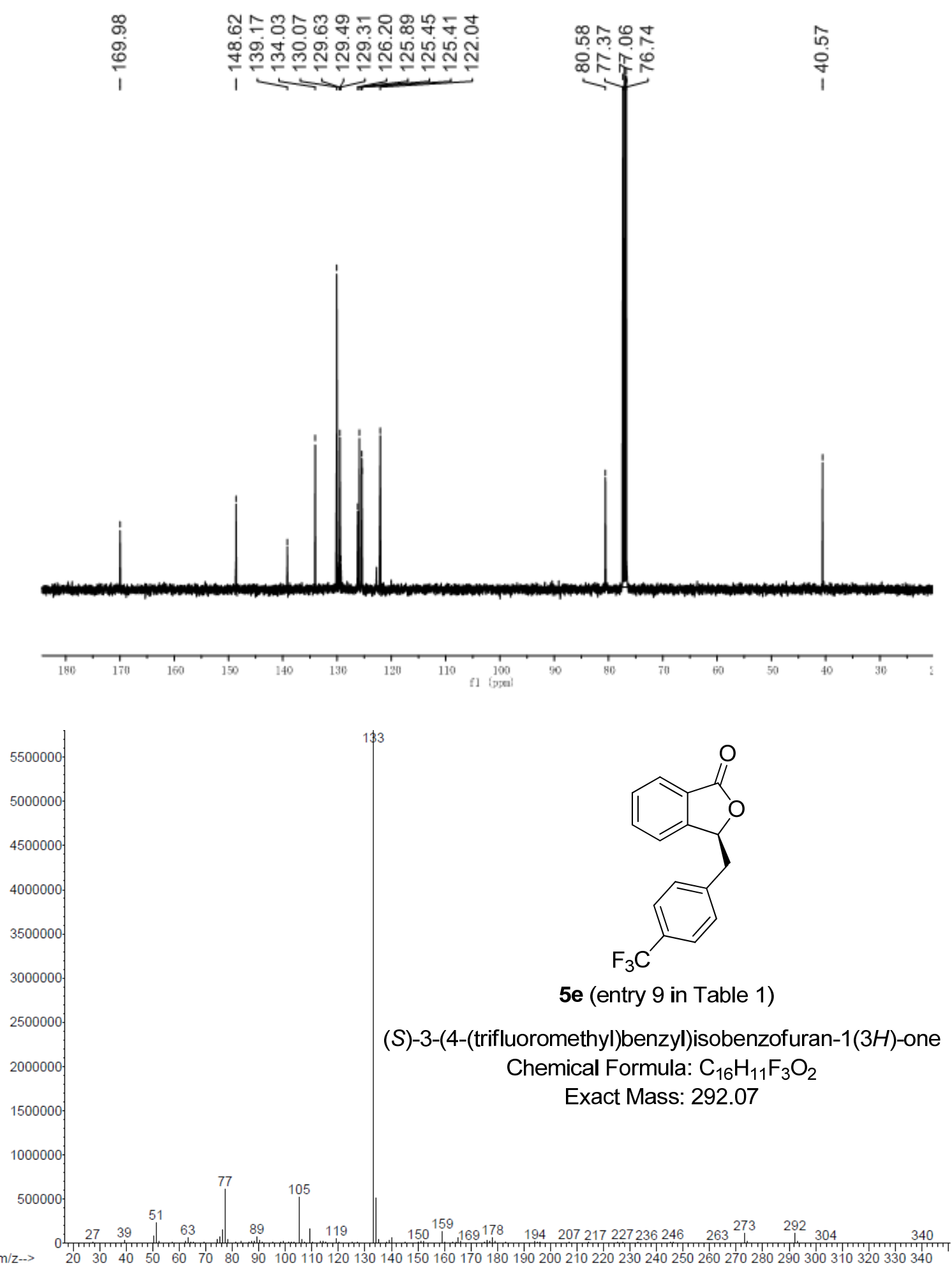
5f (entry 10 in Table 1): (S)-3-(4-methylbenzyl)isobenzofuran-1(3H)-one [Org. Lett., 2009, 11, 4712]. Yield : 97\% (99\% ee); ${ }^{1} \mathrm{H}$ NMR (400 MHz, $\left.\mathrm{CDCl}_{3}\right): \delta 2.30(\mathrm{~s}, 3 \mathrm{H})$,


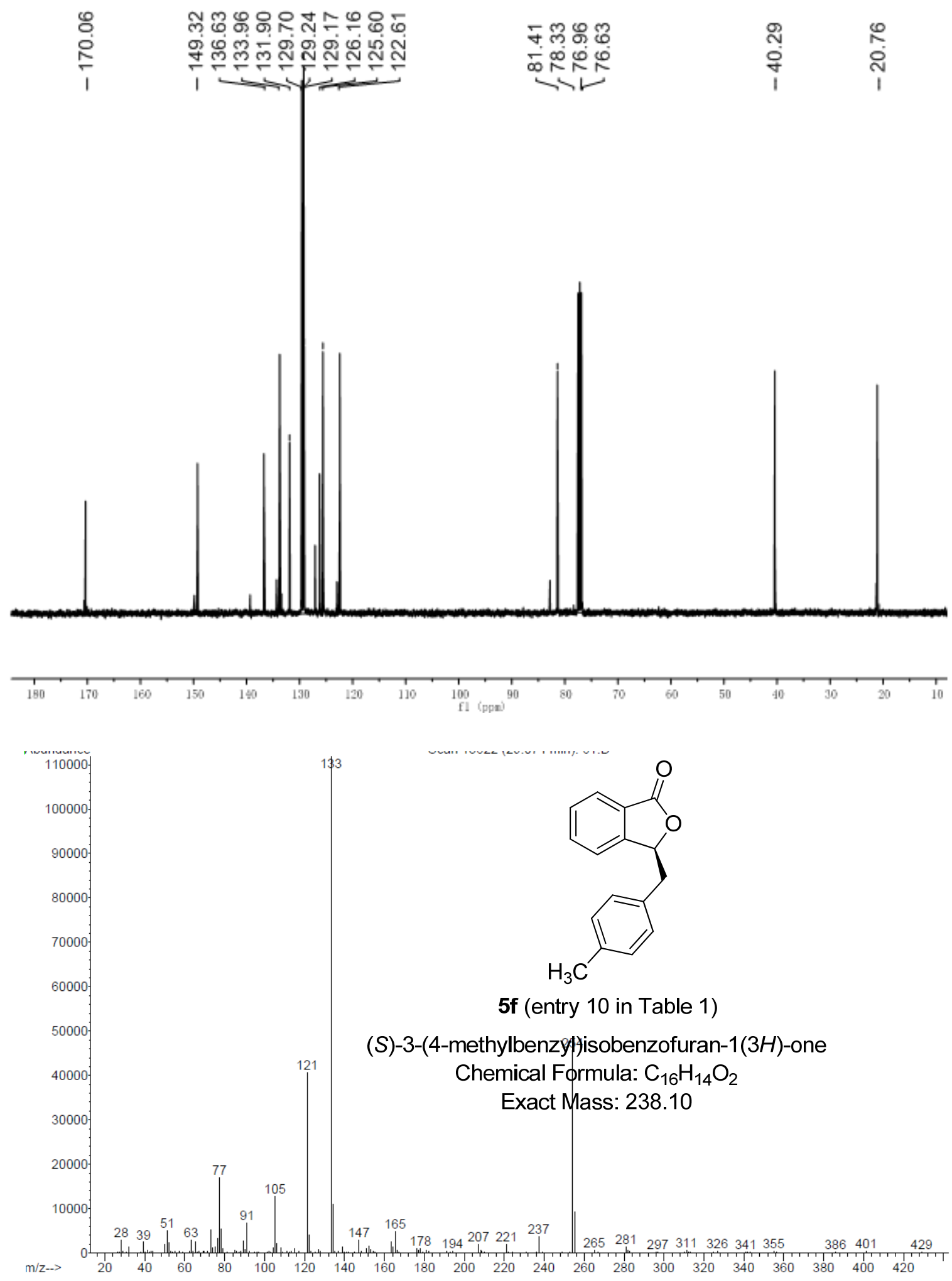
5q (entry 11 in Table 1): (S)-3-(4-methoxybenzyl)isobenzofuran-1(3H)-one [Org. Lett., 2009, 11, 4712]. Yield : 98\% (96\% ee); ${ }^{1} \mathrm{H} \mathrm{NMR}\left(400 \mathrm{MHz}, \mathrm{CDCl}_{3}\right): \delta$<smiles>COc1ccc(CC2OC(=O)c3ccccc32)cc1</smiles>
$2.97-3.03(\mathrm{dd}, J=6.4 \mathrm{~Hz}, 14 \mathrm{~Hz} 1 \mathrm{H}), 3.07-3.12(\mathrm{dd}, J=6 \mathrm{~Hz}, J=14 \mathrm{~Hz}$, $1 \mathrm{H}), 3.64(\mathrm{~s}, 3 \mathrm{H}), 5.51-5.55(\mathrm{t}, J=6.4 \mathrm{~Hz}, 1 \mathrm{H}), 6.76(\mathrm{~d}, J=8.4 \mathrm{~Hz} 2 \mathrm{H})$, $7.07(\mathrm{~d}, J=8.4 \mathrm{~Hz}, 2 \mathrm{H}), 7.17(\mathrm{~d} J=8 \mathrm{~Hz}, 1 \mathrm{H}), 7.43(\mathrm{t}, J=7.6 \mathrm{~Hz}, 1 \mathrm{H})$, $7.56(\mathrm{dt}, J=0.8 \mathrm{~Hz}, J=7.6 \mathrm{~Hz}, 1 \mathrm{H}), 7.71(\mathrm{~d}, J=7.6 \mathrm{~Hz}, 1 \mathrm{H}) .{ }^{13} \mathrm{C}\left\{{ }^{1} \mathrm{H}\right\}$ NMR (101 MHz, $\left.\mathrm{CDCl}_{3}\right): \delta 39.85,55.20,81.43,113.88,122.41,125.56$, $126.23,126.90,129.15,130.77,149.19,158.65,170.36$; GC/MS (m/z): 254.

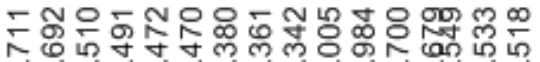

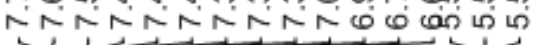<smiles>CCCC=CCCC</smiles>

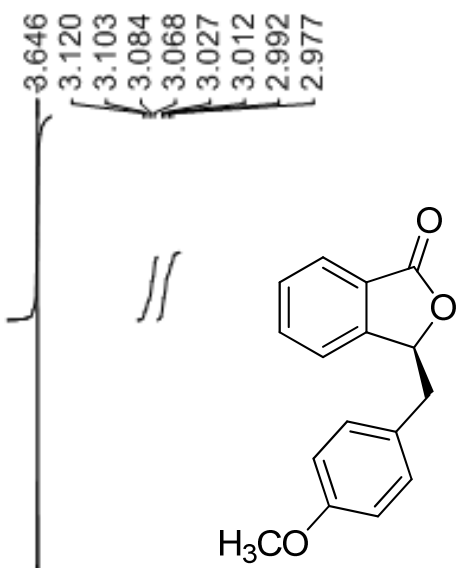

$\mathbf{5 g}$ (entry 11 in Table 1)

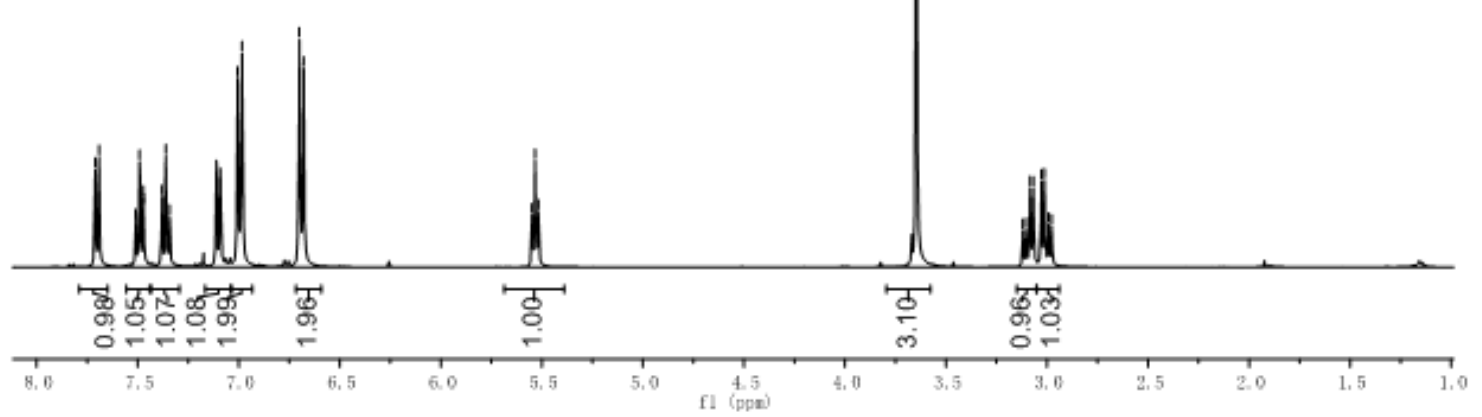



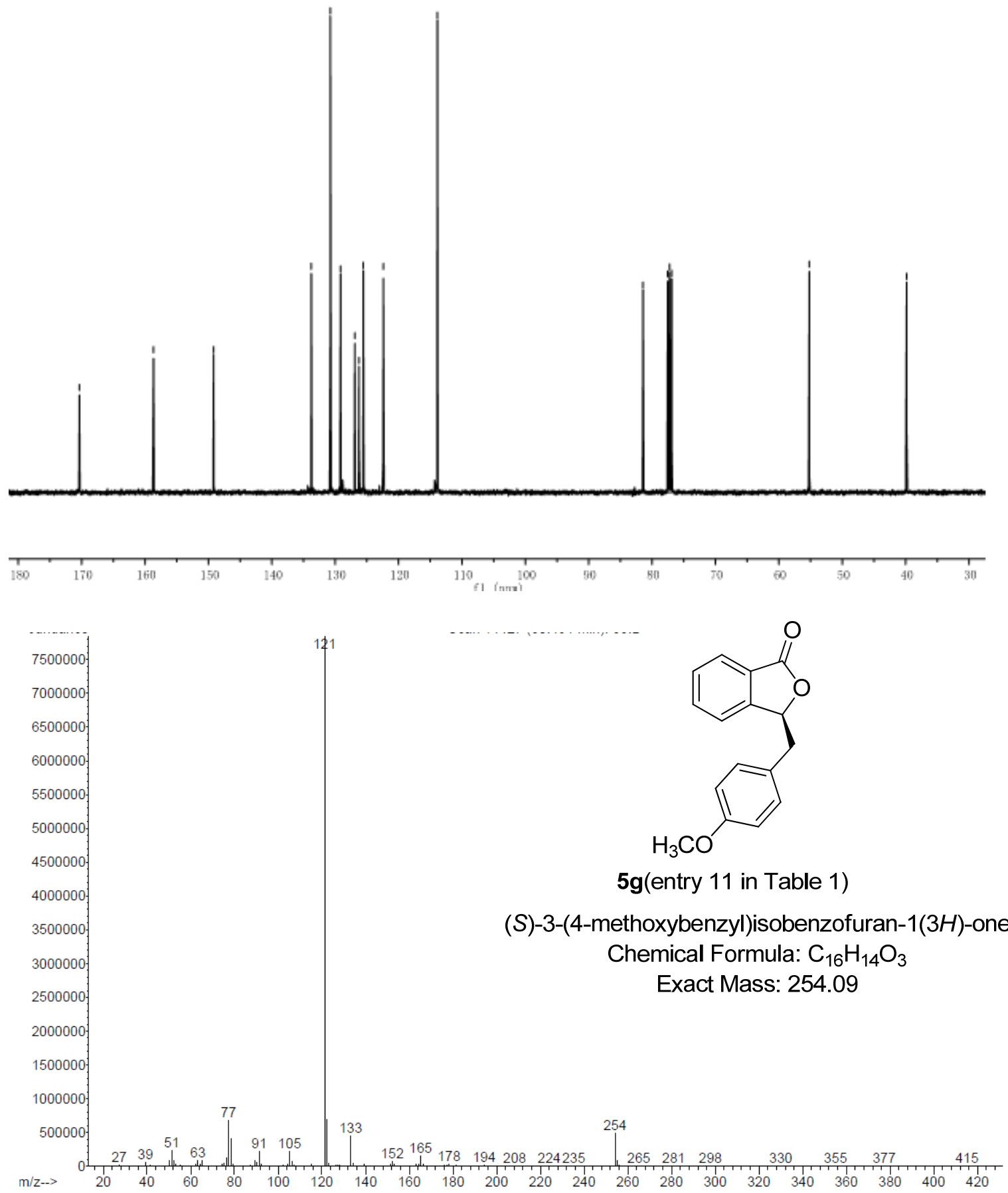
5h(entry 12 in Table 1): (S)-3-(3-methoxybenzyl)isobenzofuran-1(3H)-one [Org. Lett., 2009, 11, 4712]. Yield : 97\% (99\% ee); ${ }^{1} \mathrm{H}$ NMR (400 $\left.\mathrm{MHz}, \mathrm{CDCl}_{3}\right): \delta$ $3.10-3.15(\mathrm{dd}, J=6.4 \mathrm{~Hz}, J=14 \mathrm{~Hz} 1 \mathrm{H}), 3.23-3.28(\mathrm{dd}, J=6.4 \mathrm{~Hz}, J=14$ $\mathrm{Hz}, 1 \mathrm{H}), 3.76(\mathrm{~s}, 3 \mathrm{H}), 5.67-6.71(\mathrm{t}, J=6.4 \mathrm{~Hz}, 1 \mathrm{H}), 6.75-6.82(\mathrm{~m}, 3 \mathrm{H})$, $7.20(\mathrm{t}, J=8 \mathrm{~Hz}, 2 \mathrm{H}), 7.48(\mathrm{t}, J=7.6 \mathrm{~Hz}, 1 \mathrm{H}), 7.58(\mathrm{dt}, J=1.2 \mathrm{~Hz}, J=7.6$ $\mathrm{Hz}, 1 \mathrm{H}), 7.84(\mathrm{~d}, J=7.6 \mathrm{~Hz}, 1 \mathrm{H}) .{ }^{13} \mathrm{C}\left\{{ }^{1} \mathrm{H}\right\} \mathrm{NMR}\left(101 \mathrm{MHz}, \mathrm{CDCl}_{3}\right): \delta$ $40.87,55.22,81.15,112.67,115.29,122.04,122.39,125.67,126.23$, 129.22,129.55,133.47,136.57, 149.13, 159.67, 170.26; GC/MS (m/z): 254.

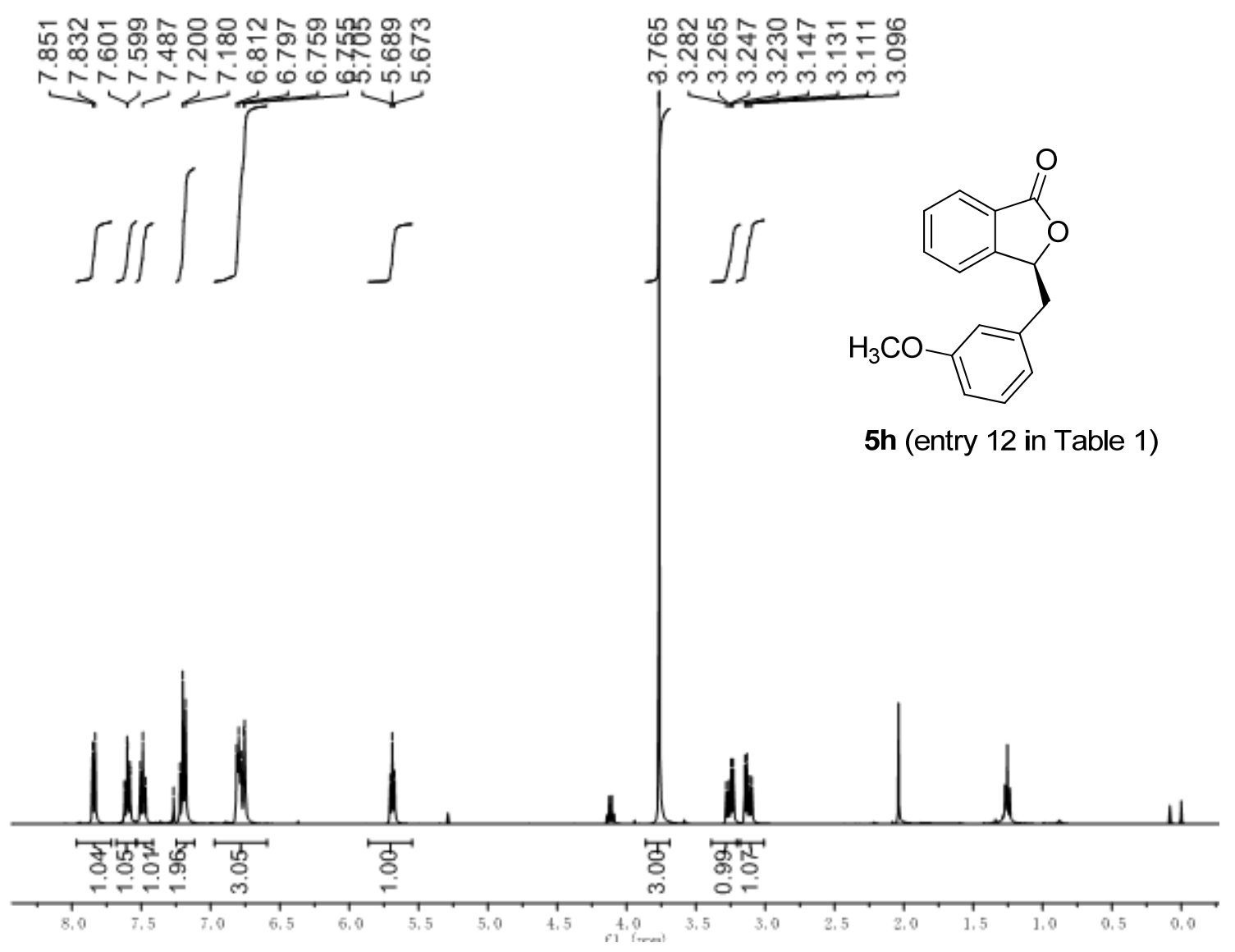



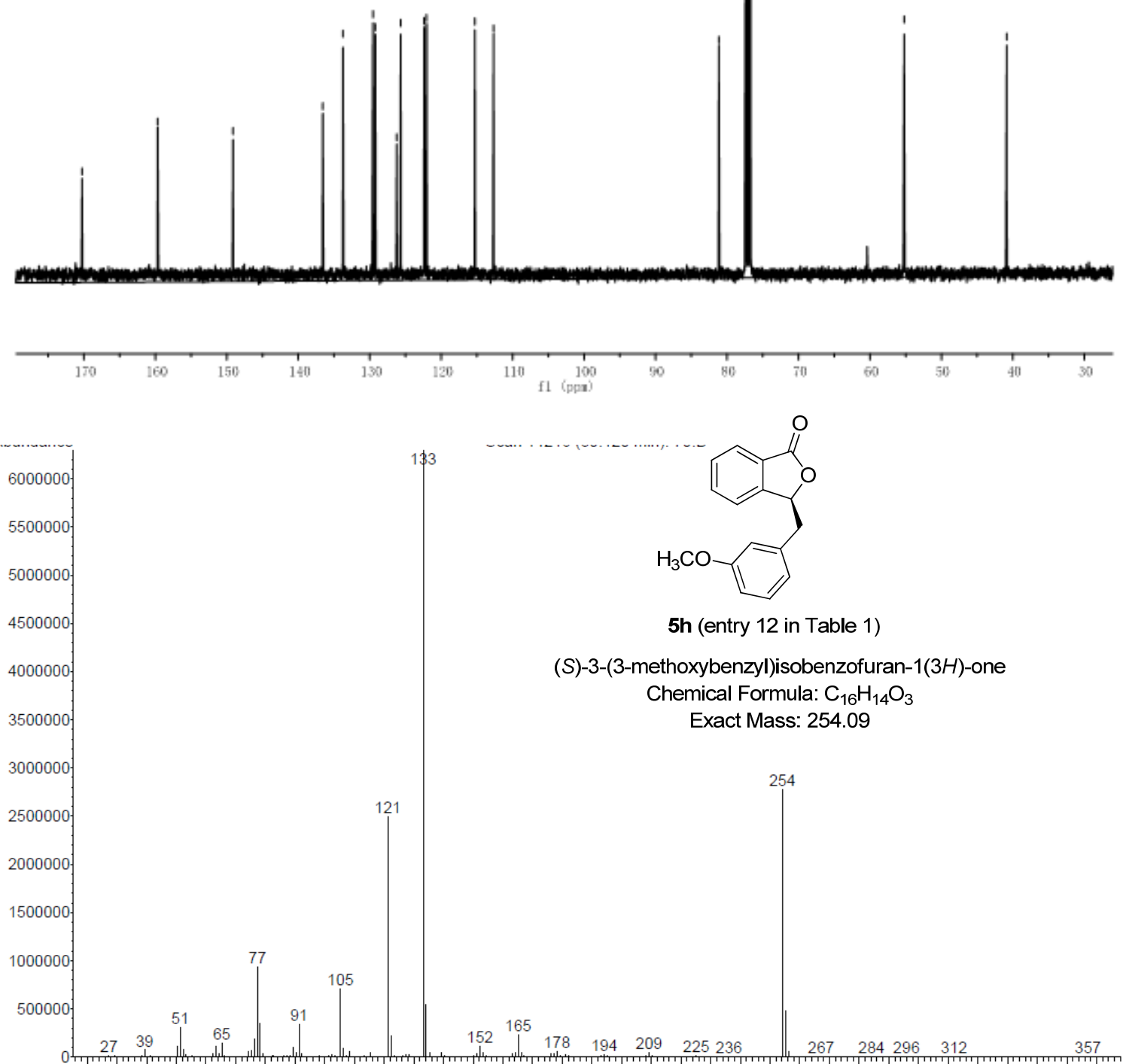
5i (entry 13 in Table 1): (S)-3-(3,4-dimethoxybenzyl)isobenzofuran-1(3H)-one [Org.<smiles>COc1ccc(CC2OC(=O)c3ccccc32)cc1OC</smiles>
Lett., 2009, 11, 4712]. Yield : 93\% (99\% ee); ${ }^{1} \mathrm{H}$ NMR (400 MHz, $\left.\mathrm{CDCl}_{3}\right): \delta 3.10-3.16(\mathrm{dd}, J=6.0 \mathrm{~Hz}, J=14 \mathrm{~Hz} 1 \mathrm{H}), 3.20-3.26(\mathrm{dd}, J=$ $6.0 \mathrm{~Hz}, J=14 \mathrm{~Hz}, 1 \mathrm{H}), 3.80(\mathrm{~s}, 3 \mathrm{H}), 3.83(\mathrm{~s}, 3 \mathrm{H}), 5.67-5.70(\mathrm{t}, J=6$ $\mathrm{Hz}, 1 \mathrm{H}), 6.68-6.78(\mathrm{~m}, 3 \mathrm{H}), 7.22(\mathrm{dd}, J=0.4 \mathrm{~Hz}, J=7.6 \mathrm{~Hz}, 1 \mathrm{H}), 7.47$ (t, $J=7.6 \mathrm{~Hz}, 1 \mathrm{H}), 7.60(\mathrm{dt}, J=1.2 \mathrm{~Hz}, J=7.6 \mathrm{~Hz}, 1 \mathrm{H}), 7.81(\mathrm{~d}, J=$ $7.6 \mathrm{~Hz}, 1 \mathrm{H}) .{ }^{13} \mathrm{C}\left\{{ }^{1} \mathrm{H}\right\}$ NMR $\left(101 \mathrm{MHz}, \mathrm{CDCl}_{3}\right): \delta 40.21,55.82,55.85$, $81.27,111.1,112.8,121.96,122.39,125.61,127.26,129.24,133.65$, 148.06, 148.71, 149.11, 170.30; GC/MS (m/z): 284.






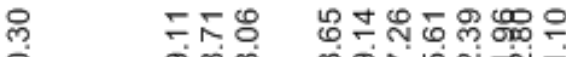

i

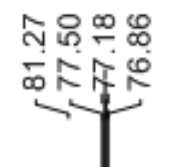

$\underbrace{\bar{n}}_{\substack{n \\ \infty}}$
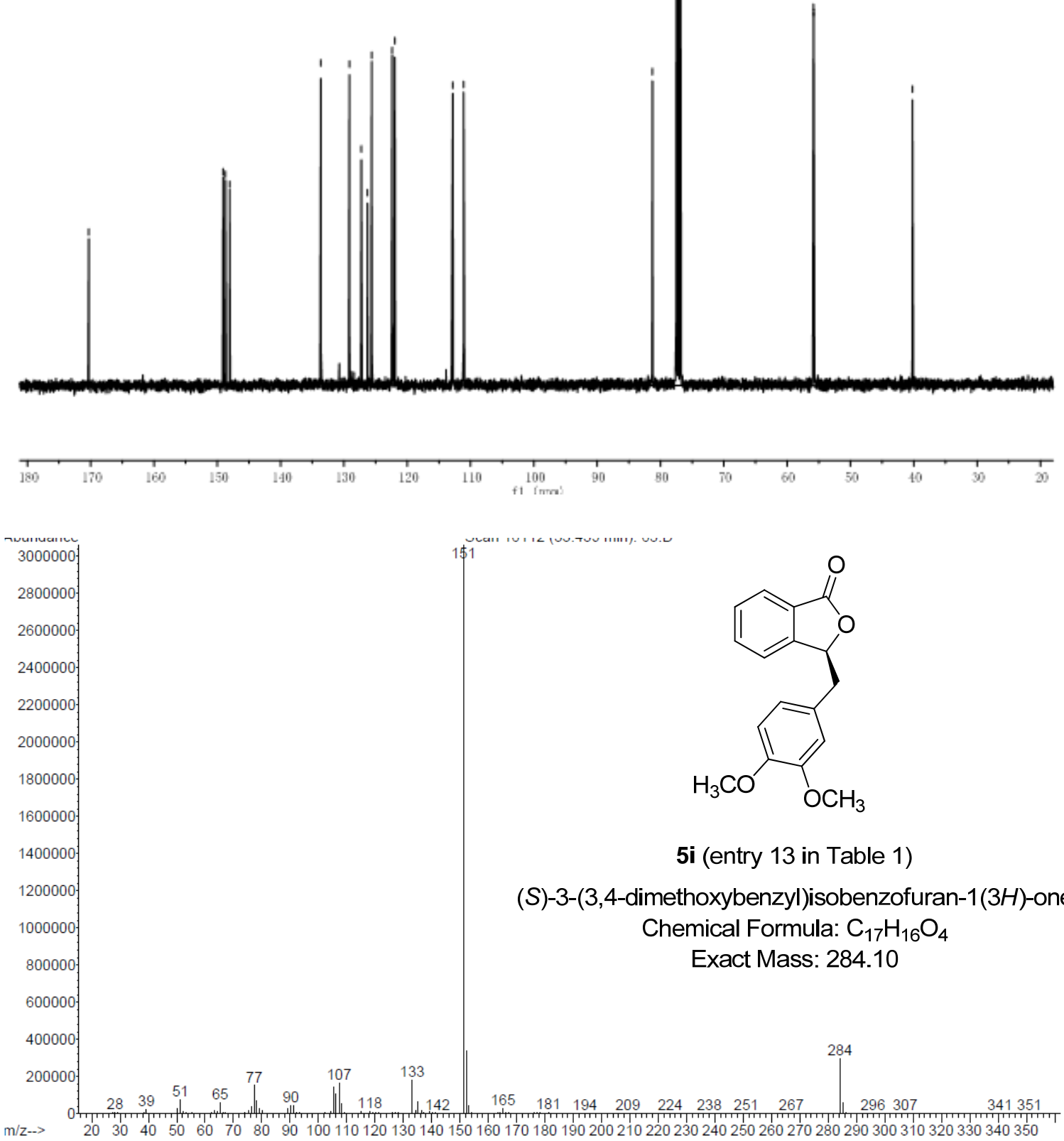
5j (entry 14 in Table 1): (S)-3-(naphthalen-1-ylmethyl)isobenzofuran-1(3H)-one [Org. Lett., 2009, 11, 4712]. Yield : 97\% (98\% ee); ${ }^{1} \mathrm{H} \mathrm{NMR}\left(400 \mathrm{MHz}, \mathrm{CDCl}_{3}\right)$ : $\delta 3.44-3.50(\mathrm{dd}, J=7.2 \mathrm{~Hz}, J=14 \mathrm{~Hz}, 1 \mathrm{H}), 3.76-3.81(\mathrm{dd}, J=7.2 \mathrm{~Hz}, J=$ $14 \mathrm{~Hz}, 1 \mathrm{H}), 5.84$ (t, $J=6.8 \mathrm{~Hz}, 1 \mathrm{H}), 6.96(\mathrm{~d}, J=7.6 \mathrm{~Hz}, 1 \mathrm{H}), 7.41-7.55$ (m, $6 \mathrm{H}), 7.82(\mathrm{~d}, J=8.4 \mathrm{~Hz}, 1 \mathrm{H}), 7.90(\mathrm{~m}, 2 \mathrm{H}), 8.08(\mathrm{~d}, J=8.4 \mathrm{~Hz}, 1 \mathrm{H})$; ${ }^{13} \mathrm{C}\left\{{ }^{1} \mathrm{H}\right\}$ NMR $\left(101 \mathrm{MHz}, \mathrm{CDCl}_{3}\right): \delta 38.40,80.47,122.74,123.27,125.43$, $125.77,125.91,126.07,126.50,128.25,128.43,129.10,129.28,131.33$, 131.90, 133.68, 133.96, 149.40, 170.25; GC/MS (m/z): 274.

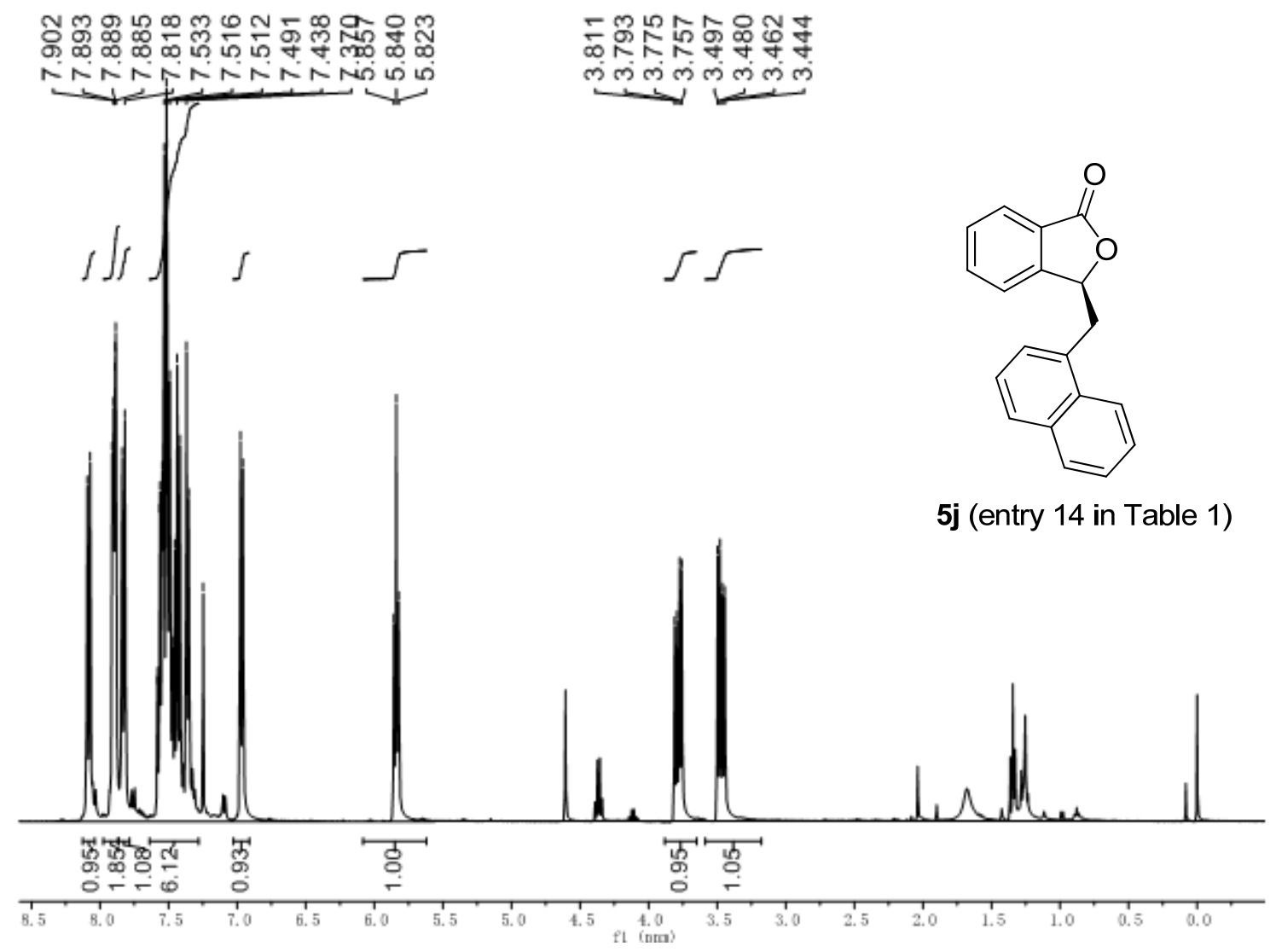



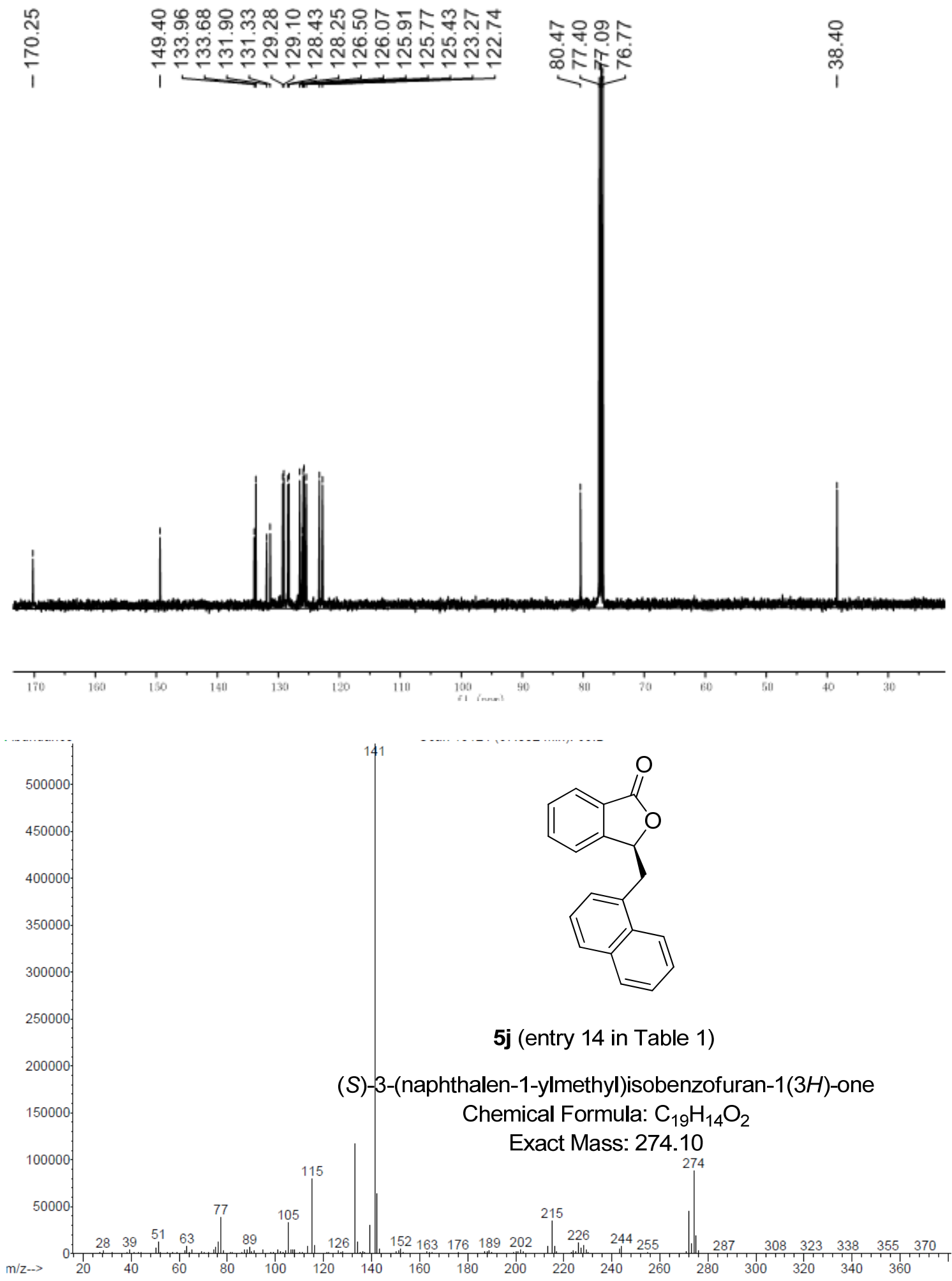
5k (entry 15 in Table 1): (S)-3-(naphthalen-2-ylmethyl)isobenzofuran-1(3H)-one [Org. Lett., 2009, 11, 4712]. Yield: 98\% (99\% ee); ${ }^{1} \mathrm{H}$ NMR (400 MHz, $\left.\mathrm{CDCl}_{3}\right): \delta 3.22-3.28$

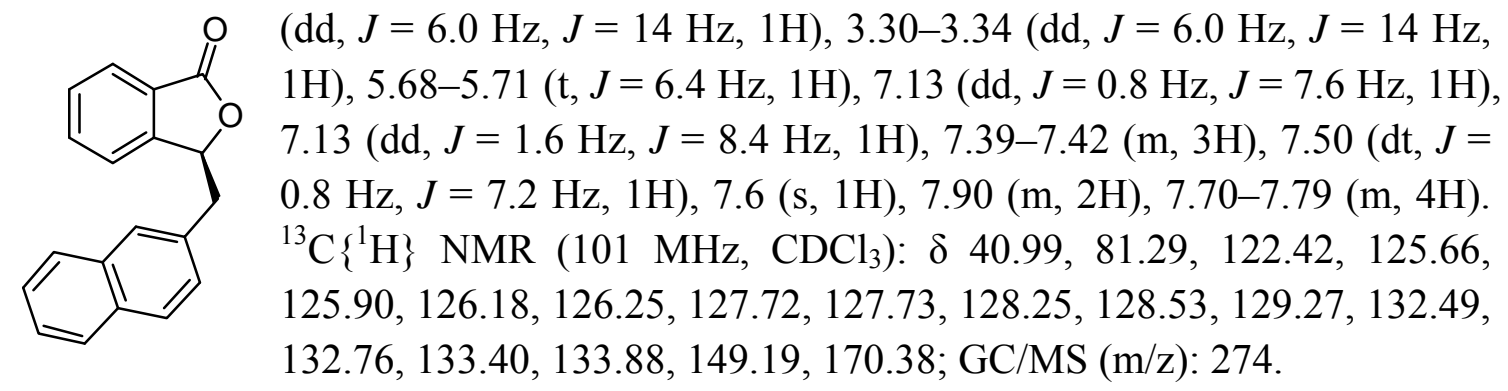

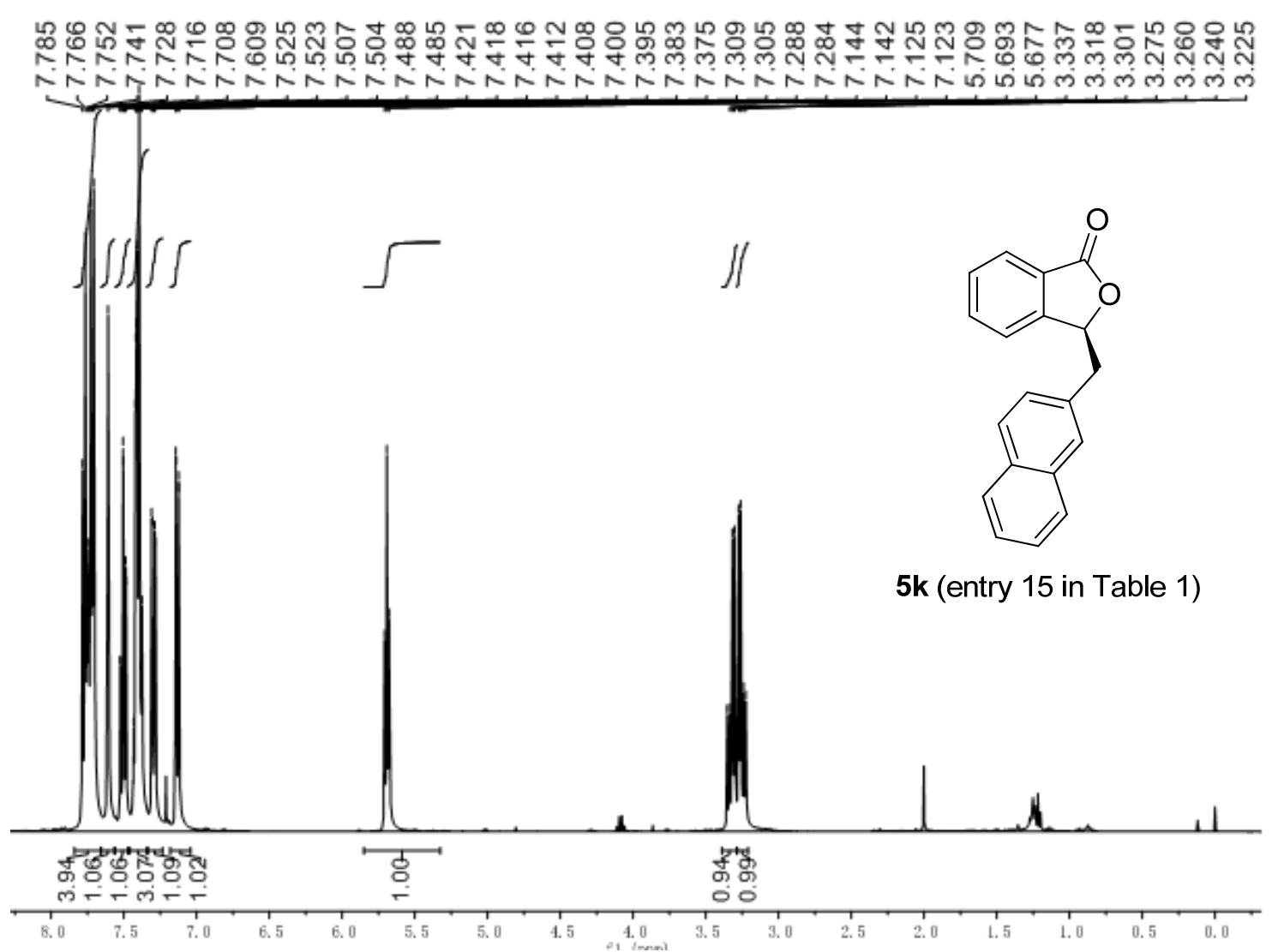



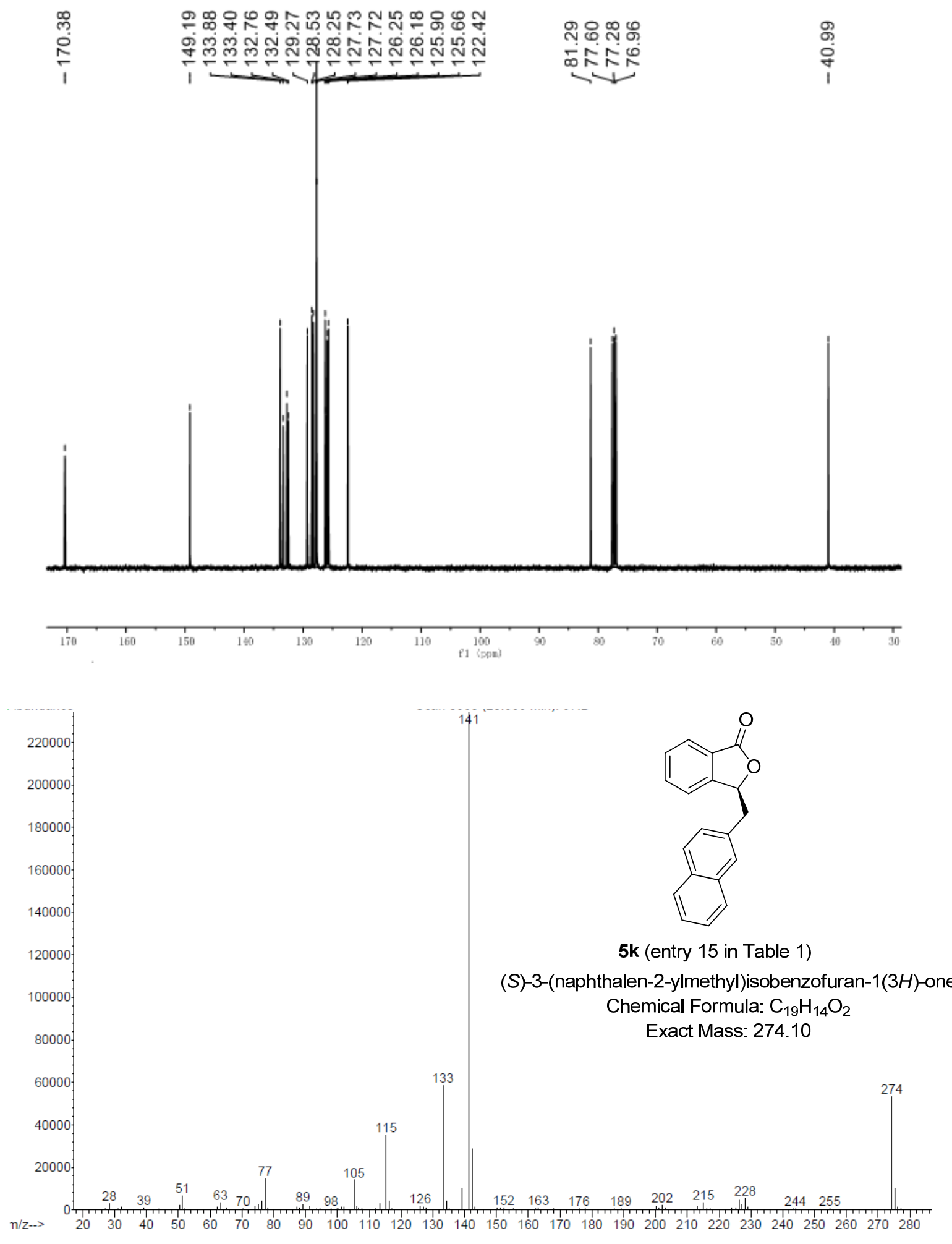

Figure S5. ${ }^{1} \mathrm{H}-\mathrm{NMR},{ }^{13} \mathrm{C}-\mathrm{NMR}$ and GC-MS of all chiral products. 
The ee values could be determined by a HPLC analysis using a Daicel chiralcel column as follows (Translation of Chinese to English is as follows).

\begin{tabular}{|c|c|c|c|c|c|c|}
\hline entry & name & $\begin{array}{c}\text { RetTime } \\
\text { [min] }\end{array}$ & peak & Area & Height & Area $\%$ \\
\hline \multicolumn{7}{|c|}{ 미化合物表视图 } \\
\hline II & 名称 & 保留时间 & 峰草 & 面皕 & 高度 & 面埥x \\
\hline 1 & RT19.833 & 19.833 & 1 & 195780 & 7923 & 1.5872 \\
\hline 2 & RT20.787 & 20.787 & 2 & 12139019 & 302479 & 88. 4128 \\
\hline
\end{tabular}

5a (entry 1 in Table 1): (S)-3-benzylisobenzofuran-1(3H)-one (HPLC: AD-H, $254 \mathrm{~nm}, \mathrm{n}-$ hexane : 2-propanol $=95: 5$, flow rate $=0.7 \mathrm{~mL} / \mathrm{min})$. [Literature (Org. Lett., 2009, 11, 4712). HPLC: ChiracelAD-H, eluent: $n$-hexane/i-propanol $=95 / 5$, flow rate $=0.7$ $\mathrm{mL} / \mathrm{min}$, detected at $254 \mathrm{~nm}$, Retention time:20.3 $\min (\mathrm{R}), 21.3 \mathrm{~min}(\mathrm{~S})]$.

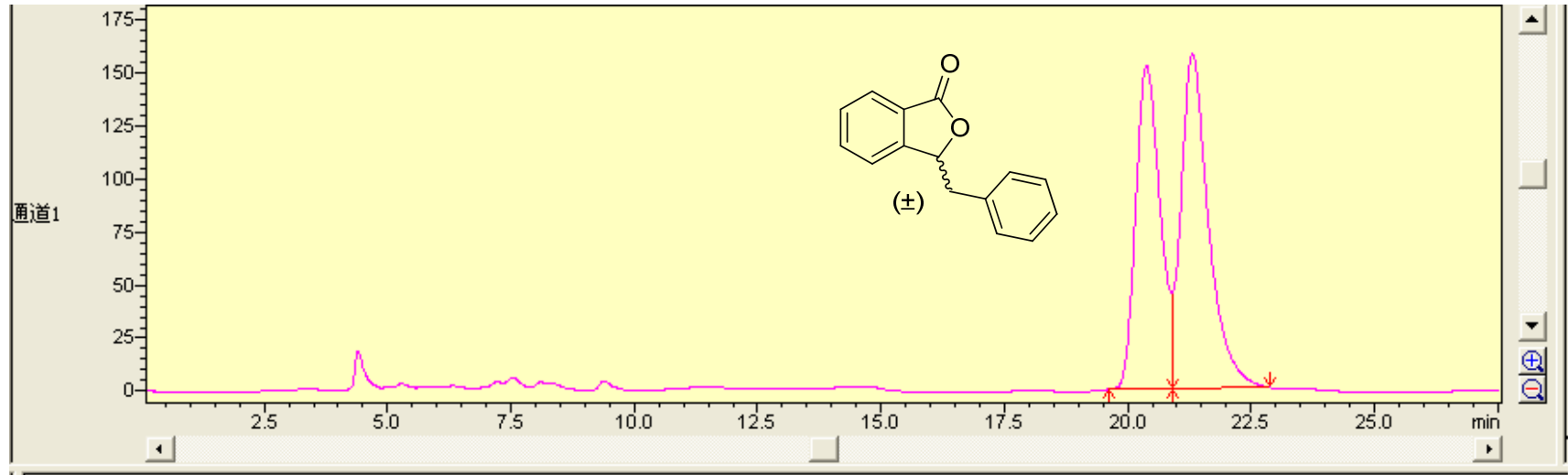

\begin{tabular}{|c|c|c|c|c|c|c|}
\hline ID* & 名称 & 保留时间 & 峰部 & 面呮 & 高度 & 面趴x \\
\hline 1 & RT20.379 & 20.379 & 1 & 5225809 & 152026 & 45.8968 \\
\hline 2 & RT21. 309 & 21.309 & 2 & 6160201 & 157858 & 54.1032 \\
\hline
\end{tabular}

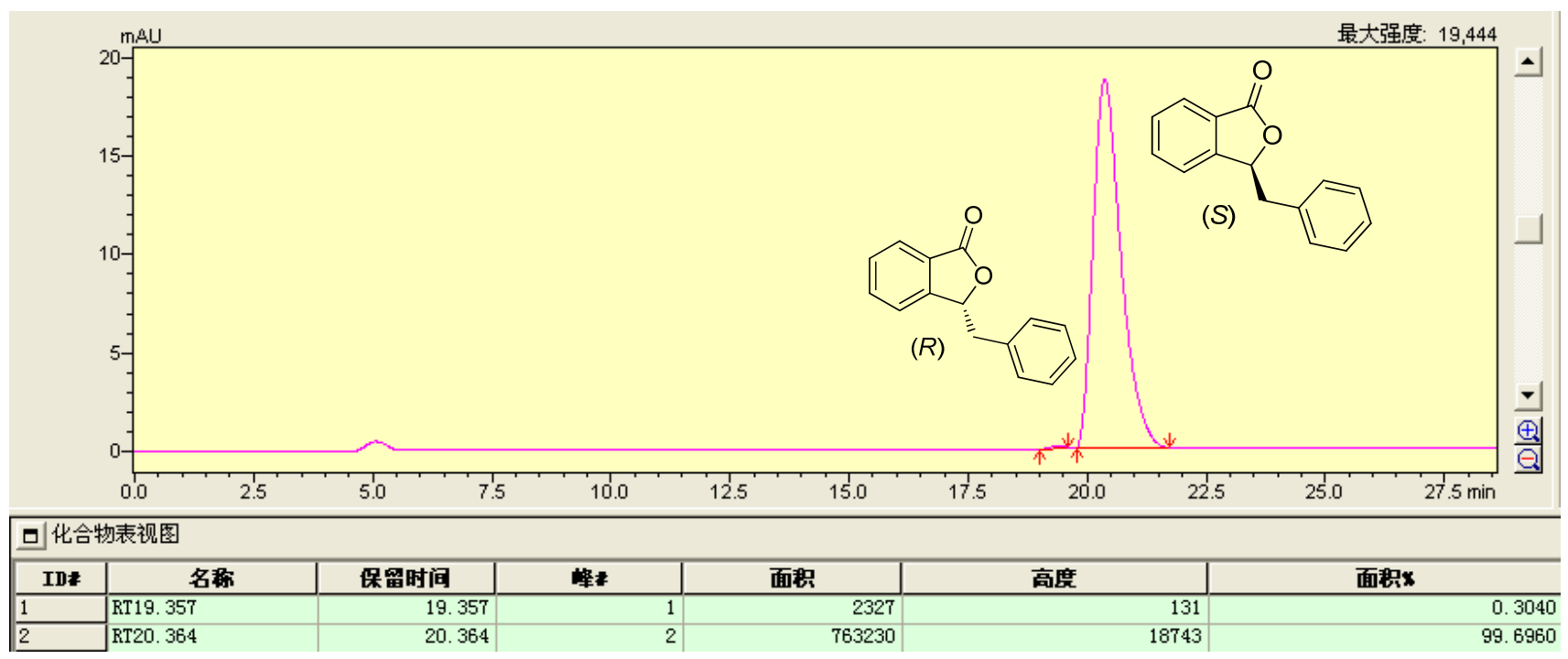


5 b (entry 6 in Table 1): (S)-3-(4-fluorobenzyl)isobenzofuran-1(3H)-one (HPLC: OB-H, $254 \mathrm{~nm}$, hexane : 2-propanol $=80: 20$, flow rate $1.0 \mathrm{~mL} / \mathrm{min})$.

5c (entry 7 in Table 1): (S)-3-(4-chlorobenzyl)isobenzofuran-1(3H)-one (HPLC: OB-H, $215 \mathrm{~nm}$, n-hexane : 2-propanol = $80: 20$, flow rate $1.0 \mathrm{~mL} / \mathrm{min}$ ). [Literature (Org. Lett., 2009, 11, 4712). HPLC: Chiracel OB-H Column;detected at 214 nm; n-hexane/i-propanol $=8 / 2$; flow $=1.0 \mathrm{~mL} / \mathrm{min}$; Retention time: $17.5 \mathrm{~min}(\mathrm{~S}), 20.3 \mathrm{~min}(\mathrm{R})]$.
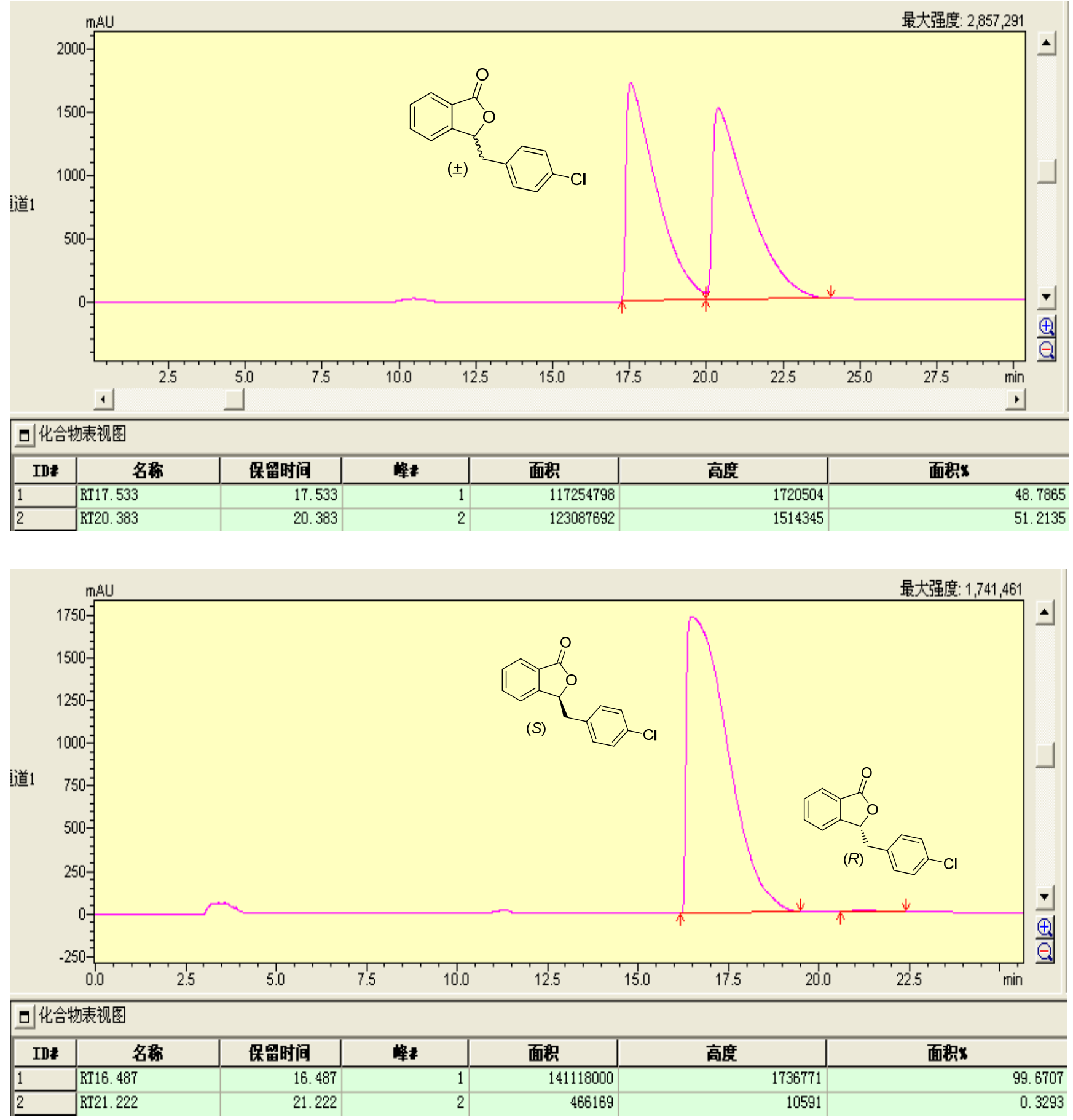
5d (entry 8 in Table 1): (S)-3-(2-chlorobenzyl)isobenzofuran-1(3H)-one (HPLC: OJ-H, $254 \mathrm{~nm}$, hexane : 2-propanol $=95: 5$,flow rate $0.7 \mathrm{~mL} / \mathrm{min}$ ).
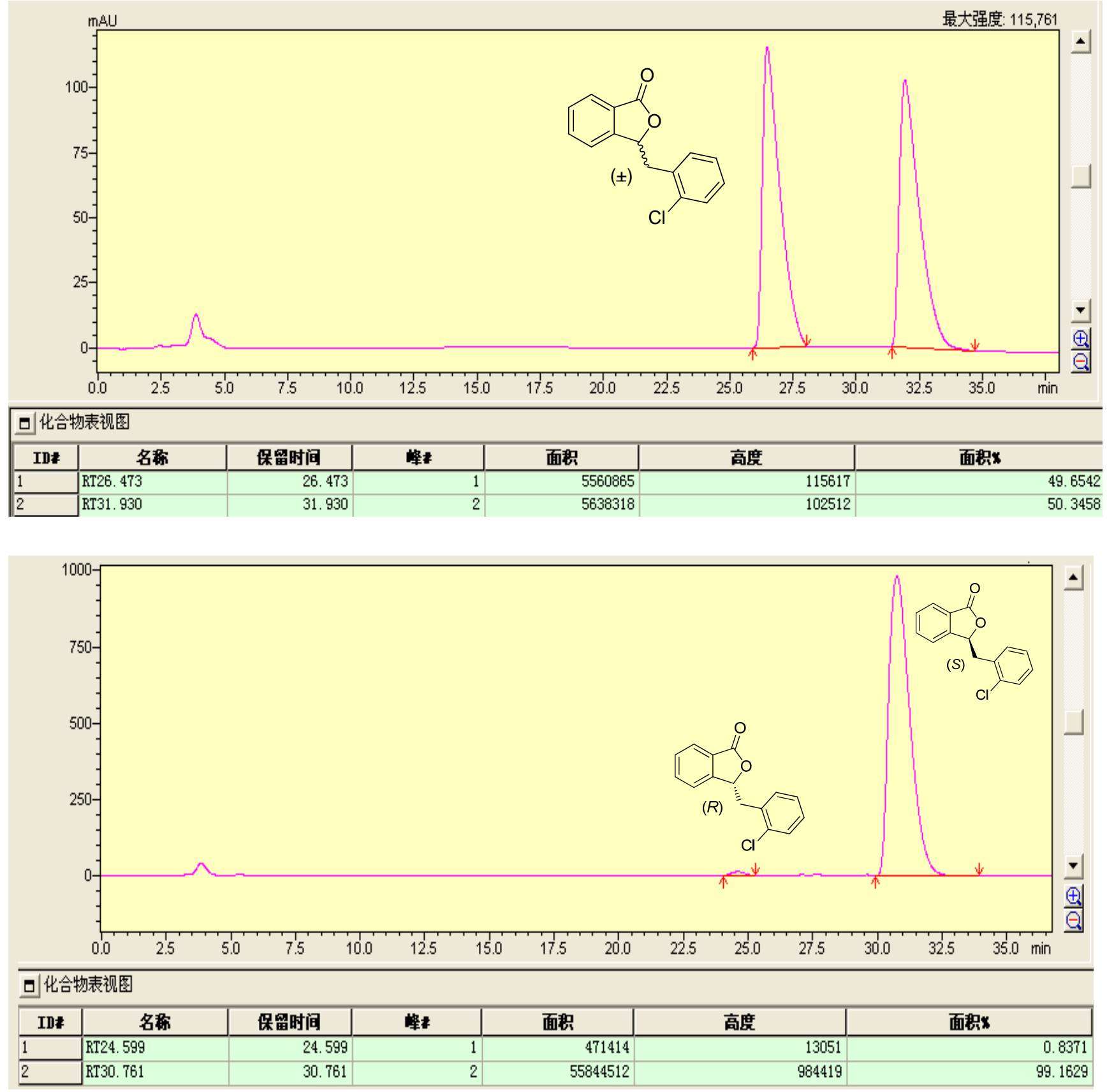
5e (entry 9 in Table 1): (S)-3-(4-(trifluoromethyl)benzyl)isobenzofuran-1(3H)-one (HPLC: OJ-H, $254 \mathrm{~nm}$, hexane : 2-propanol=90:10, flow rate $1.0 \mathrm{~mL} / \mathrm{min}$ ). [Literature (Org. Lett., 2009, 11, 4712). HPLC: Chiracel OJ-HColumn; detected at $214 \mathrm{~nm}$; nhexane $/$ i-propanol =9/1; flow $=1.0 \mathrm{~mL} / \mathrm{min}$; Retention time: $19.3 \mathrm{~min}(\mathrm{~S}), 22.4 \mathrm{~min}(\mathrm{R})]$.
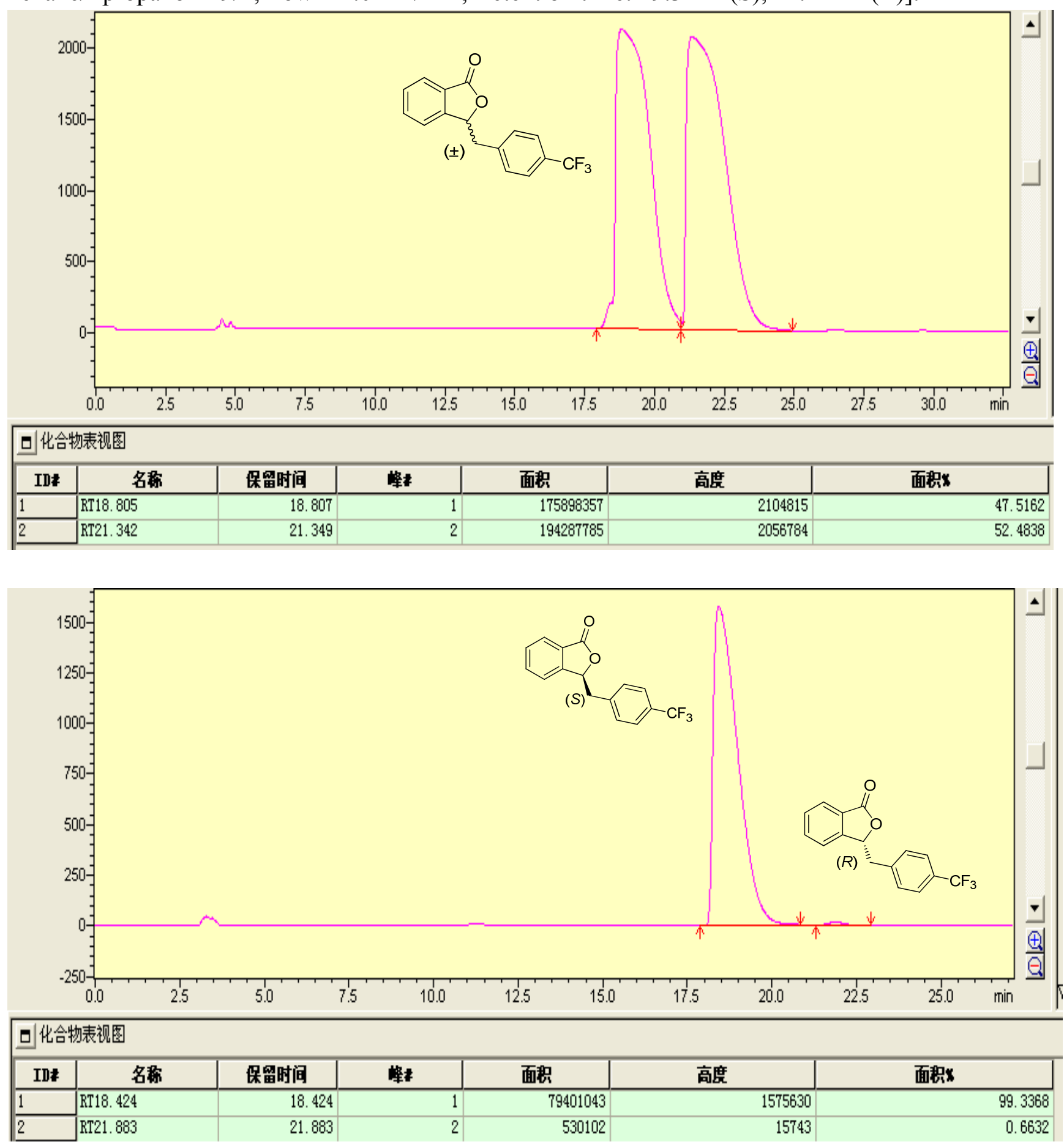
5f (entry 10 in Table 1): (S)-3-(4-methylbenzyl)isobenzofuran-1(3H)-one (HPLC: OJ-H, $254 \mathrm{~nm}$, hexane : 2-propanol $=90: 10$,flow rate $1.0 \mathrm{~mL} / \mathrm{min}$ ). [Literature (Org. Lett., 2009, 11, 4712). HPLC: Chiracel OJ-H Column;detected at $214 \mathrm{~nm}$; n-hexane / ipropanol $=9 / 1$; flow $=1.0 \mathrm{~mL} / \mathrm{min}$; Retention time: $17.6 \mathrm{~min}(\mathrm{R}), 26.5 \mathrm{~min}(\mathrm{~S})]$.
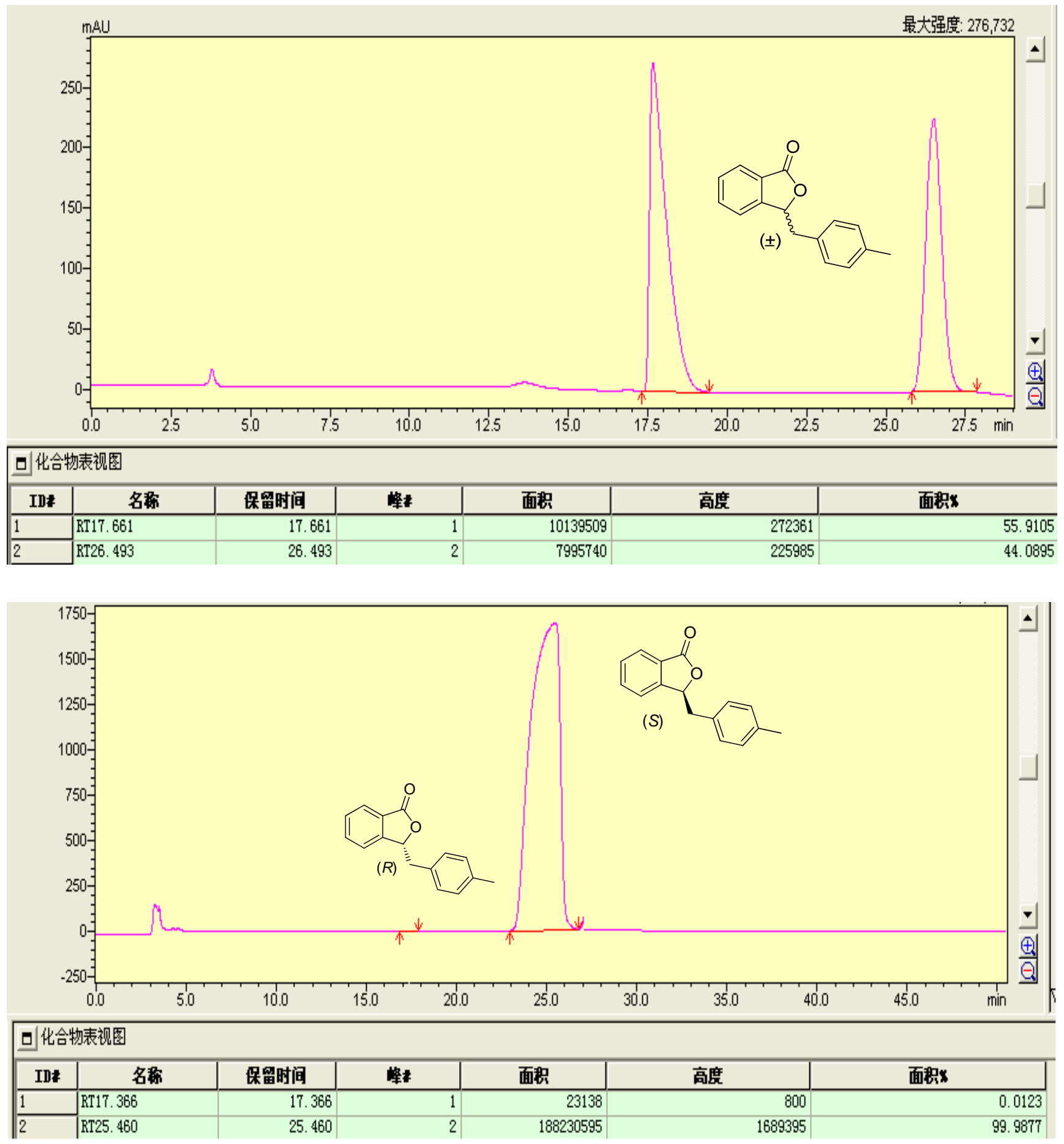
5q (entry 11 in Table 1): (S)-3-(4-methoxybenzyl)isobenzofuran-1(3H)-one (HPLC: OBH, $254 \mathrm{~nm}$, n-hexane : 2-propanol $=70: 30$, flow rate $1.0 \mathrm{~mL} / \mathrm{min}$ ). [Literature (Org. Lett., 2009, 11, 4712). HPLC: Chiracel OB-HColumn; detected at $214 \mathrm{~nm}$; n-hexane/i-propanol $=7 / 3$; flow $=1.0 \mathrm{~mL} / \mathrm{min}$; Retention time: $18.1 \mathrm{~min}(\mathrm{~S}), 25.5 \mathrm{~min}(\mathrm{R})]$
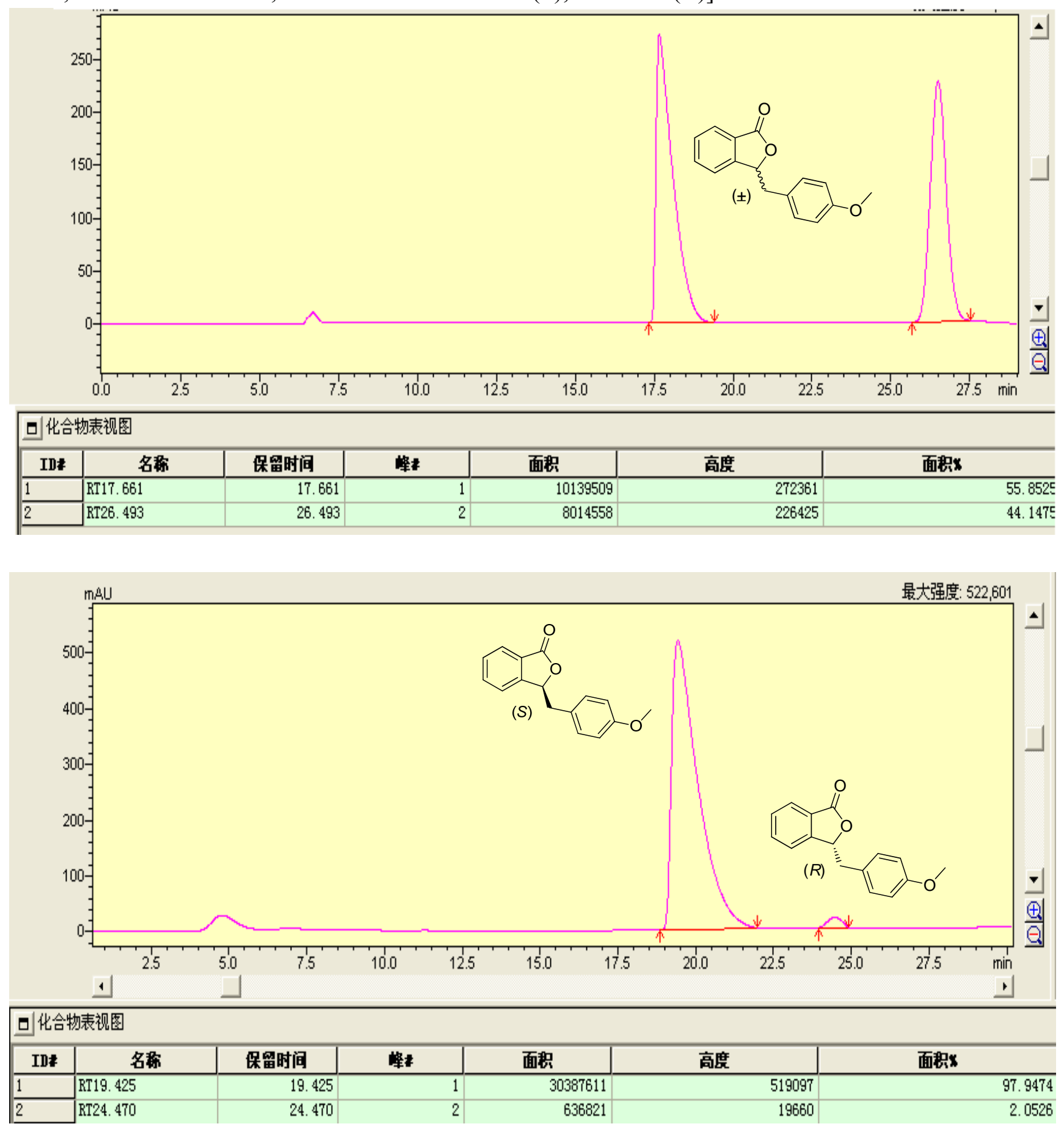
5 h (entry 12 in Table 1): (S)-3-(3-methoxybenzyl)isobenzofuran-1(3H)-one (HPLC: OB$\mathrm{H}, 254 \mathrm{~nm}$, hexane : 2-propanol $=80: 20$, flow rate $1.0 \mathrm{~mL} / \mathrm{min}$ ).
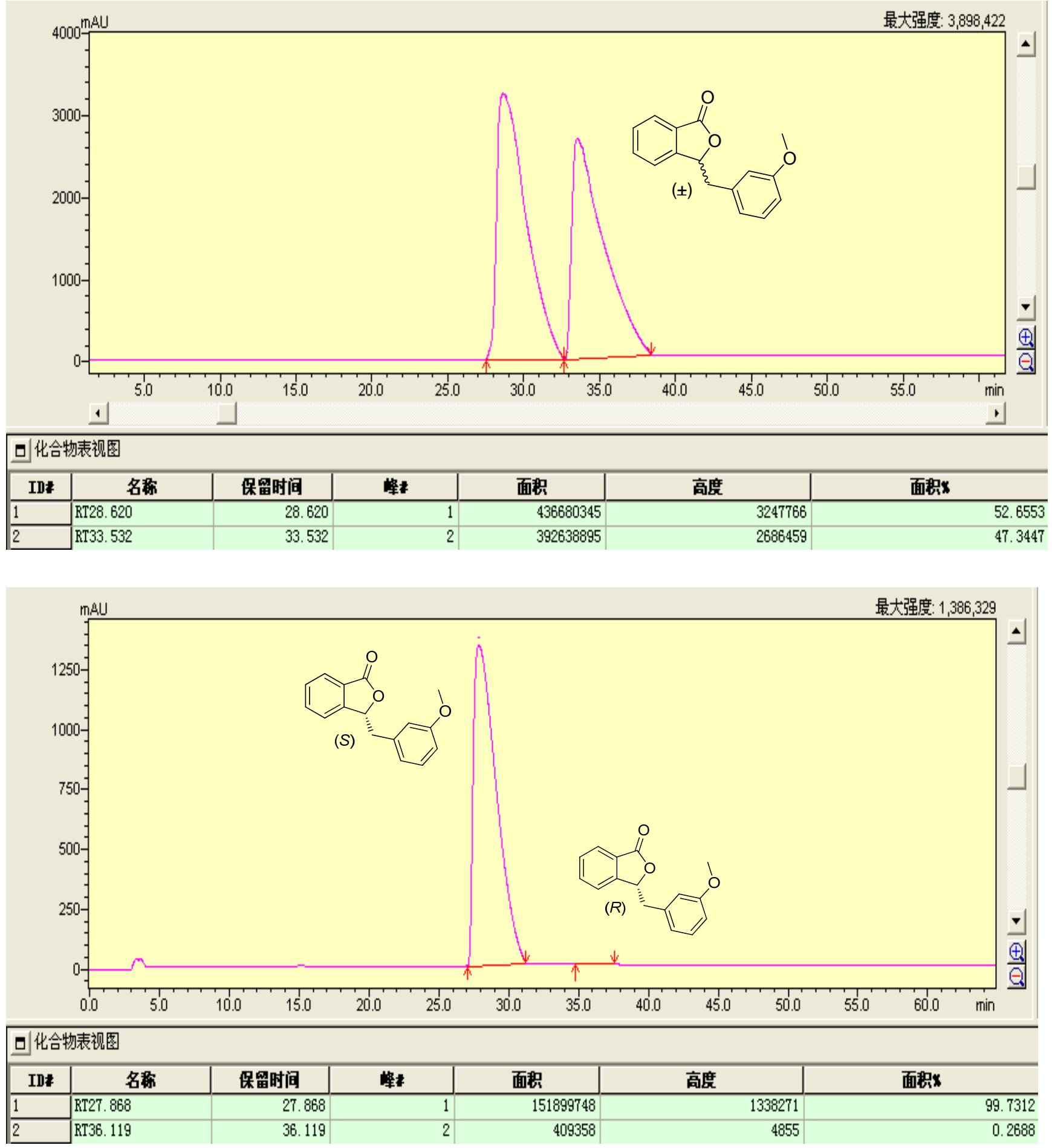
5i (entry 13 in Table 1): (S)-3-(3,4-dimethoxybenzyl)isobenzofuran-1(3H)-one (HPLC: OD-H, $254 \mathrm{~nm}$, n-hexane : 2-propanol $=80: 20$, flow rate $1.0 \mathrm{~mL} / \mathrm{min})$. [Literature (Org. Lett., 2009, 11, 4712). HPLC: Chiracel OD-HColumn; detected at $214 \mathrm{~nm}$; n-hexane/ipropanol $=8 / 2$; flow $=1.0 \mathrm{~mL} / \mathrm{min}$; Retention time: $14.9 \mathrm{~min}(\mathrm{~S}), 17.5 \mathrm{~min}(\mathrm{R})]$.
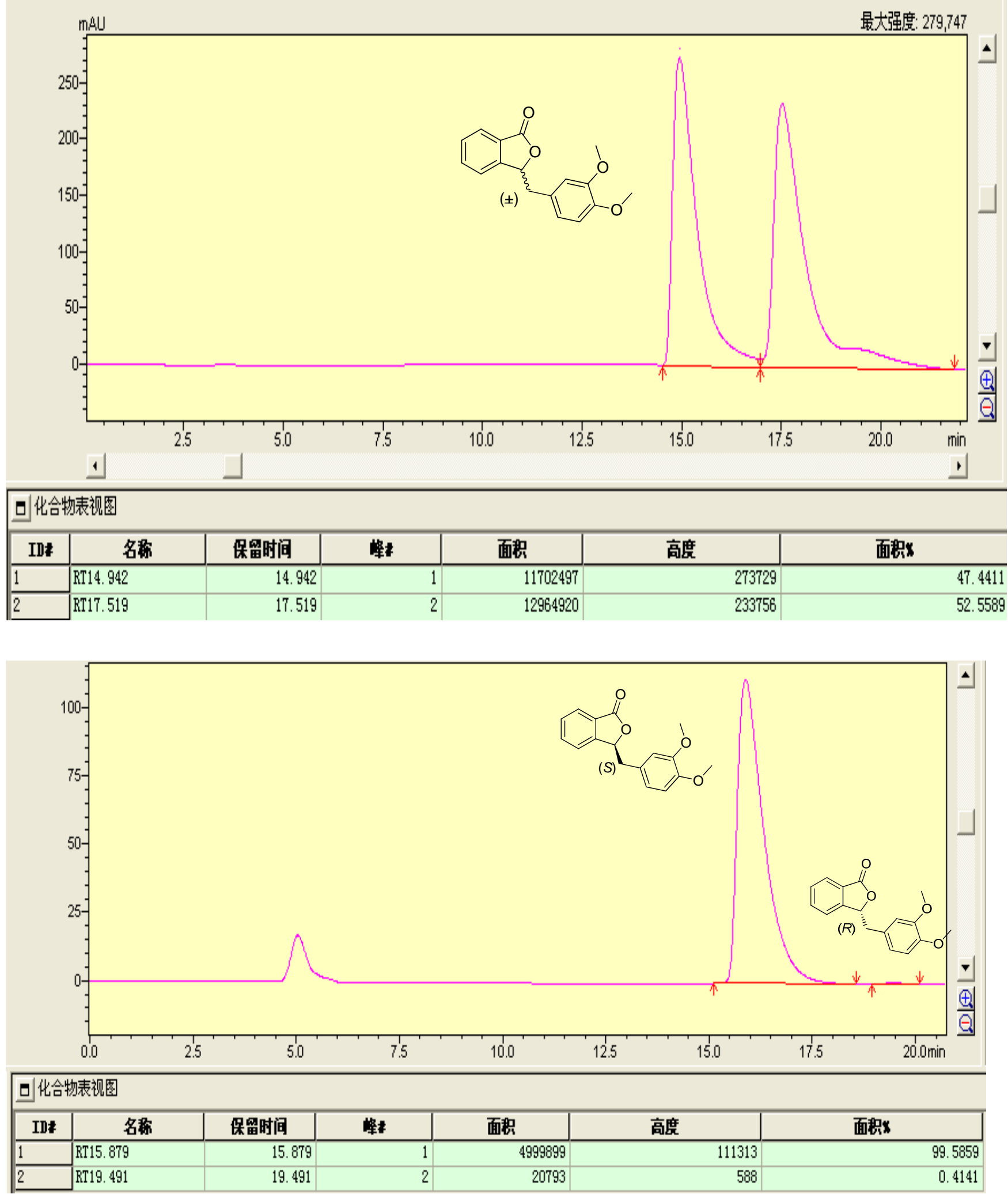
5j (entry 14 in Table 1): (S)-3-(naphthalen-1-ylmethyl)isobenzofuran-1(3H)-one (HPLC: AD-H, $254 \mathrm{~nm}$, n-hexane : 2-propanol= $90: 10$, flow rate $1.0 \mathrm{~mL} / \mathrm{min})$. [Literature (Org. Lett., 2009, 11, 4712). HPLC:Chiracel AD-HColumn (250 mm); detected at $214 \mathrm{~nm}$; nhexane $/$ i-propanol $=9 / 1$; flow $=1.0 \mathrm{~mL} / \mathrm{min}$; Retention time: $10.8 \mathrm{~min}(\mathrm{R}), 12.0 \mathrm{~min}(\mathrm{~S})$.
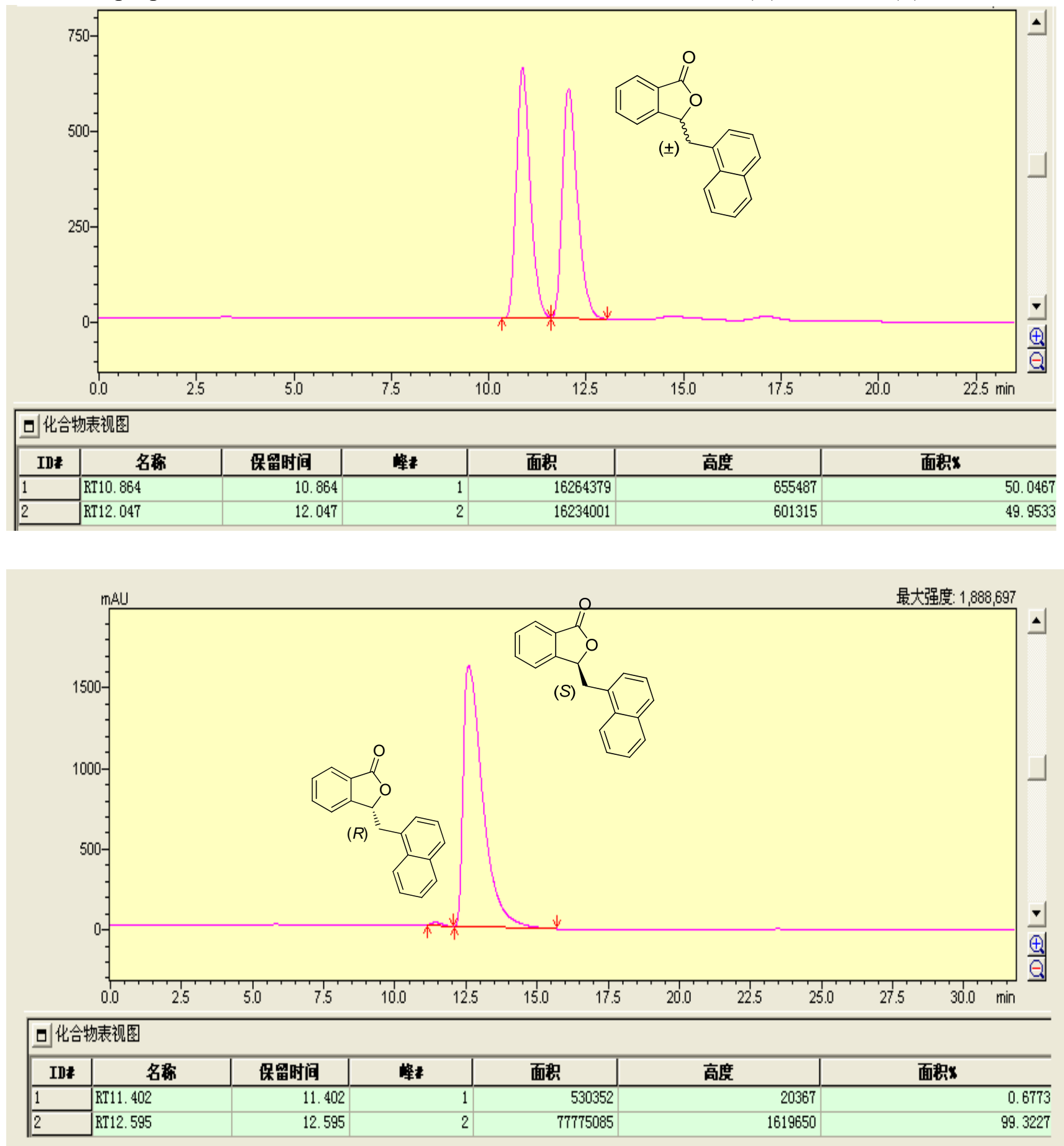
5k (entry 15 in Table 1): (S)-3-(naphthalen-2-ylmethyl)isobenzofuran-1(3H)-one (HPLC:

$\mathrm{AD}-\mathrm{H}, 254 \mathrm{~nm}$, hexane : 2-propanol $=98: 2$, flow rate $1.0 \mathrm{~mL} / \mathrm{min}$ ).
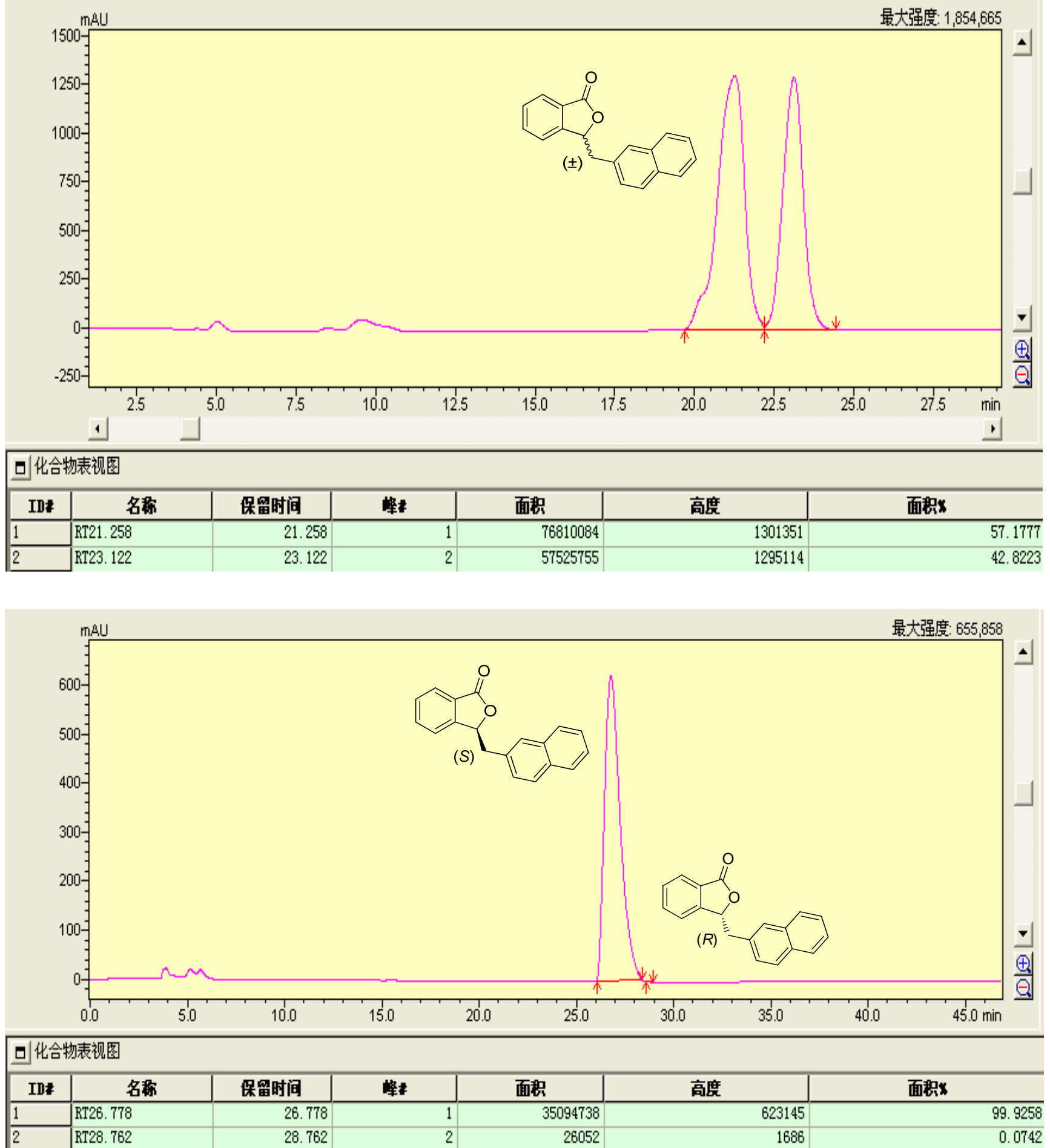

Figure S6. Asymmetric synthesis of phthalides in the enantioselectivite tandem reduction-lactonization reaction of 2-acylarylcarboxylates. [The ee value could be determined by a HPLC analysis with a Photo-Diode Array detector using a Daicel chiralcel column $(\Phi 0.46 \times 25 \mathrm{~cm})$.]. 
Table S1. Reusability of 3 using ethyl 2-(2-(4-bromophenyl)acetyl)benzoate as a substrate. $^{[\mathrm{a}]}$

\begin{tabular}{|c|c|c|c|c|c|c|c|c|}
\hline Entry & 1 & 2 & 3 & 4 & 5 & 6 & 7 & 8 \\
\hline $\begin{array}{l}\text { Yield } \\
\text { [\%] }\end{array}$ & 99 & 97 & 99 & 98 & 97 & 96 & 95 & 91 \\
\hline $\begin{array}{l}\text { ee } \\
{[\%]^{[b]}}\end{array}$ & 99 & 99 & 99 & 99 & 99 & 99 & 98 & 97 \\
\hline
\end{tabular}

Recycling experiment part: Recycle 1 .

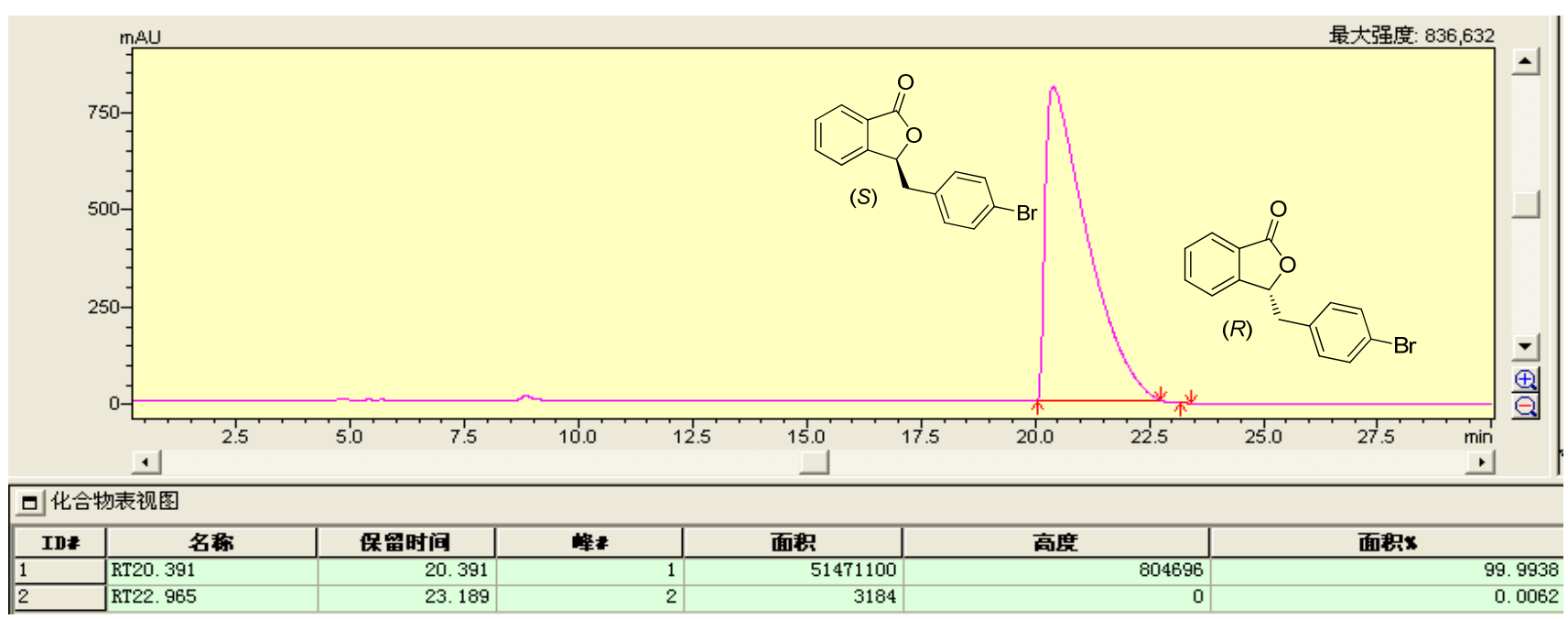

Recycle 2 .

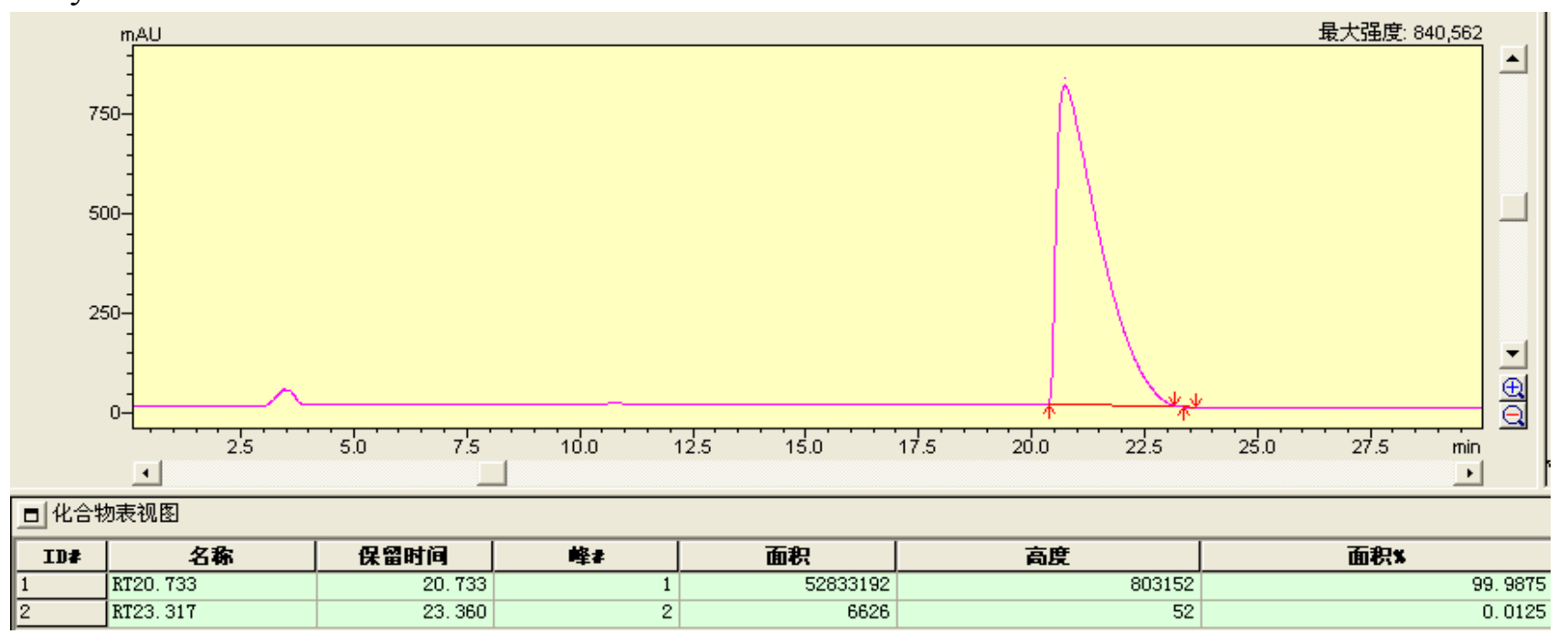


Recycle 3.

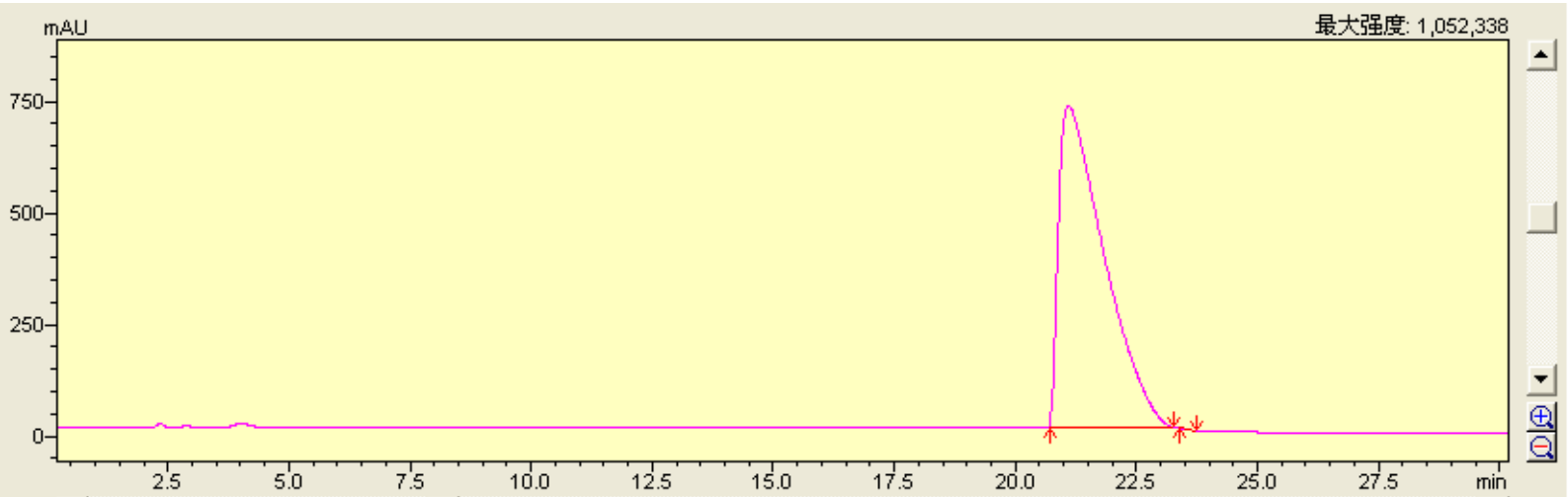

1

\begin{tabular}{|c|c|c|c|c|c|c|}
\hline \multicolumn{7}{|c|}{ 므化合物表视图 } \\
\hline ID* & 名称 & 保留时间 & 峰* & 面积 & 高度 & 面呮 $\mathrm{x}$ \\
\hline 1 & RT21.092 & 21.092 & 1 & 47026075 & 720254 & 99.9431 \\
\hline 2 & RT23. 136 & 23. 381 & 2 & 26778 & 125 & 0.0569 \\
\hline
\end{tabular}

Recycle 4.

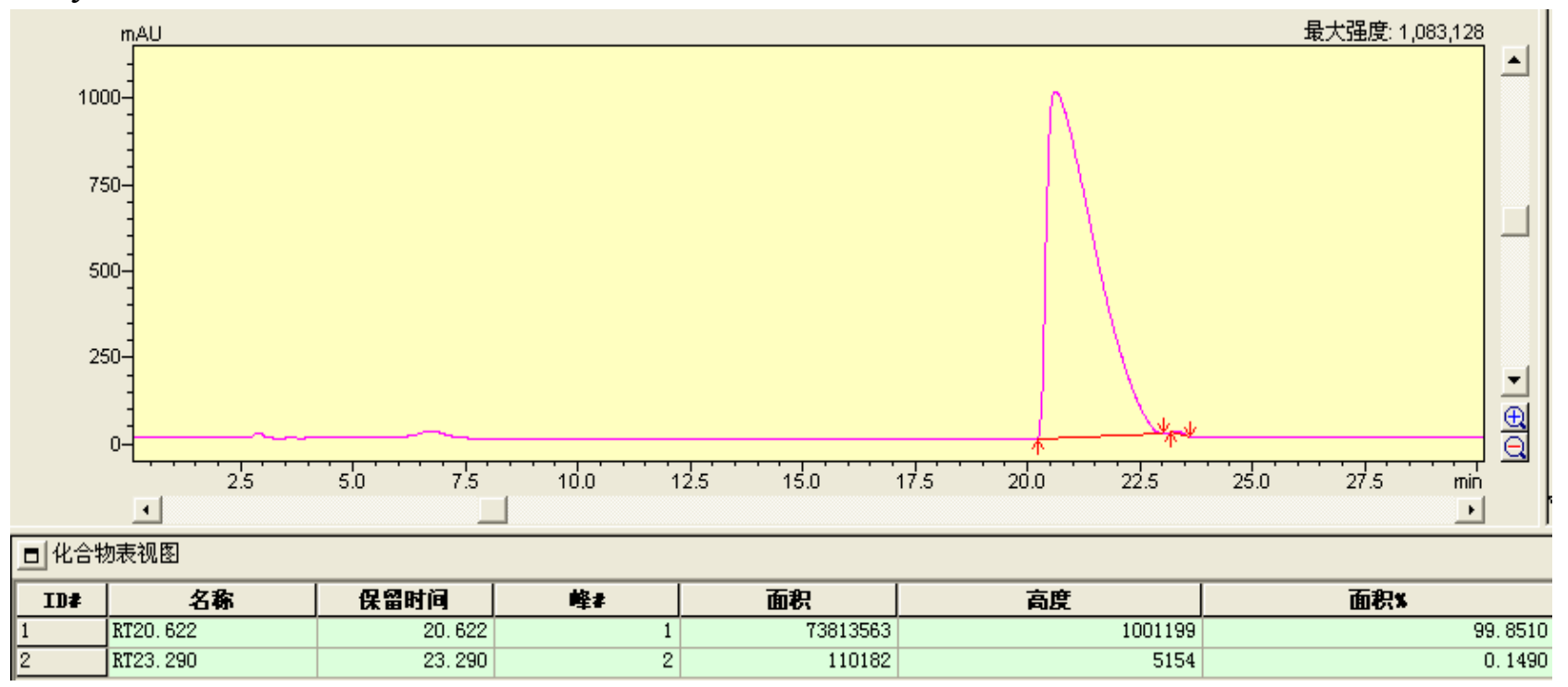

Recycle 5.



\begin{tabular}{|c|c|c|c|c|c|c|}
\hline \multicolumn{7}{|c|}{ 口化合物表视图 } \\
\hline ID* & 名称 & 保留时间 & 婥春 & 面积 & 高度 & 面积 $x$ \\
\hline 1 & RT20.901 & 20.901 & 1 & 51737893 & 797520 & 99.8400 \\
\hline 2 & RT23.367 & 23. 367 & 2 & 82939 & 4269 & 0.1600 \\
\hline
\end{tabular}


Recycle 6.



\begin{tabular}{|c|c|c|c|c|c|c|}
\hline IDE & 名称 & 足勾时间 & 峰 & 面吿 & 高席 & 面那义 \\
\hline 1 & RT21.116 & 21.116 & 1 & 55128717 & 810308 & 99.9219 \\
\hline 2 & RT23. 584 & 23.424 & 2 & 43105 & 88 & 0.0781 \\
\hline
\end{tabular}

Recycle 7.

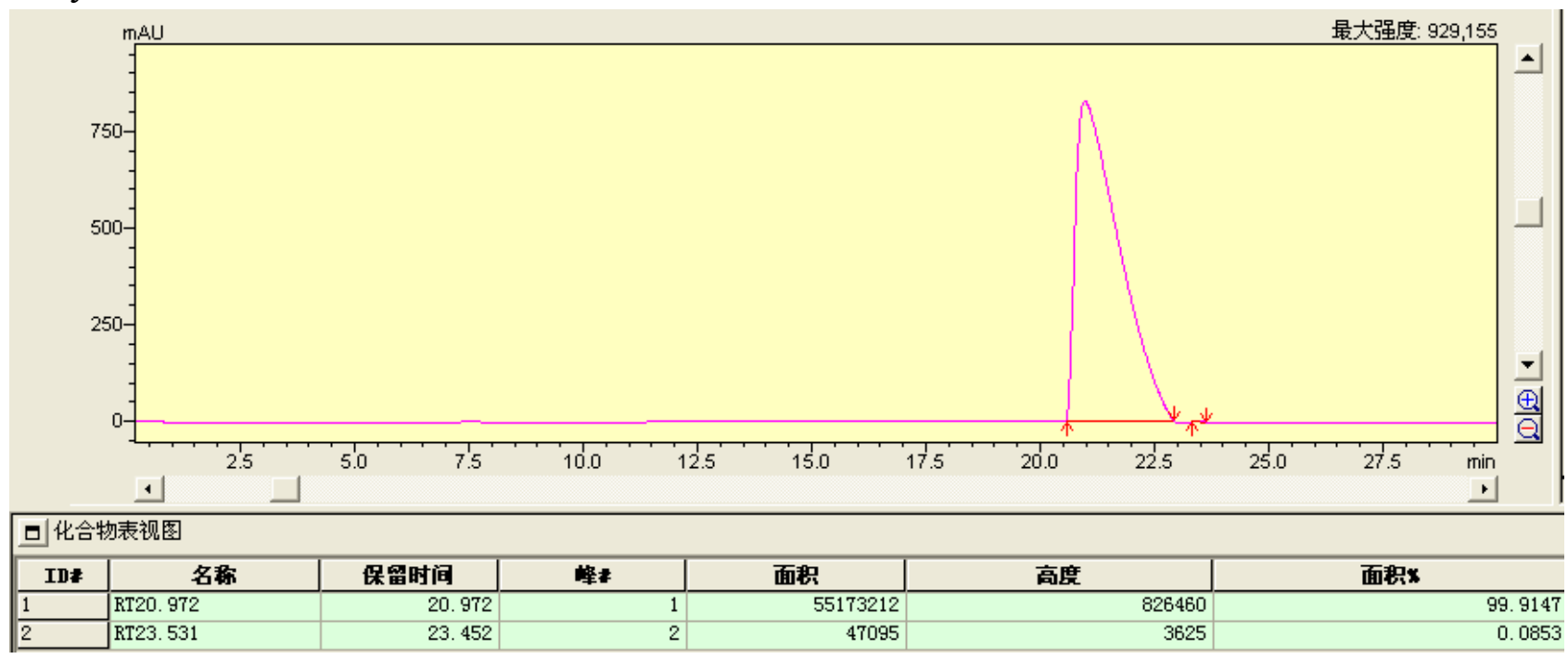

Recycle 8.

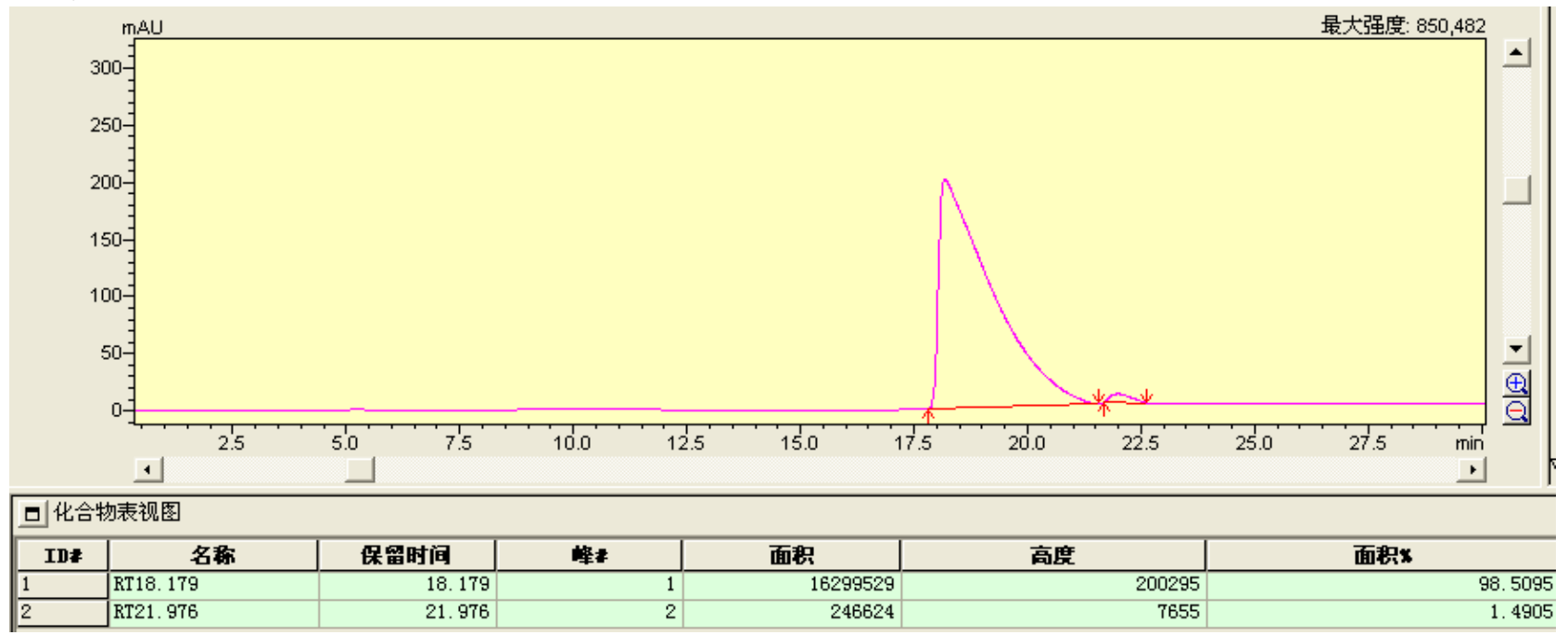

Figure S7. Reusability of 3 using ethyl 2-(2-(4-bromophenyl)acetyl)benzoate as a substrate. 